\title{
Transverse measures, the modular class, and a cohomology pairing for Lie algebroids
}

\author{
Sam Evens and Jiang-Hua Lu \\ Department of Mathematics, University of Arizona, Tucson, AZ 85721 USA \\ Alan Weinstein \\ Department of Mathematics, University of California, Berkeley, CA 94720 USA
}

May 28, 2018

\begin{abstract}
We show that every Lie algebroid $A$ over a manifold $P$ has a natural representation on the line bundle $Q_{A}=\wedge^{t o p} A \otimes \wedge^{t o p} T^{*} P$. The line bundle $Q_{A}$ may be viewed as the Lie algebroid analog of the orientation bundle in topology, and sections of $Q_{A}$ may be viewed as transverse measures to $A$. As a consequence, there is a well-defined class in the first Lie algebroid cohomology $H^{1}(A)$ called the modular class of the Lie algebroid $A$. This is the same as the one introduced earlier by Weinstein using the Poisson structure on $A^{*}$. We show that there is a natural pairing between the Lie algebroid cohomology spaces of $A$ with trivial coefficients and with coefficients in $Q_{A}$. This generalizes the pairing used in the Poincare duality of finite-dimensional Lie algebra cohomology. The case of holomorphic Lie algebroids is also discussed, where the existence of the modular class is connected with the Chern class of the line bundle $Q_{A}$.
\end{abstract}

\section{Contents}

\begin{tabular}{lll}
\hline 1 & Introduction & 2
\end{tabular}

$\begin{array}{|ll|}2 & \text { Differential calculus on Lie algebroids }\end{array}$

\begin{tabular}{|lll}
\hline 3 & The representation of $A$ on $Q_{A}$ and the modular class of $A$ & 7
\end{tabular}

\begin{tabular}{|lll}
\hline 4 & The case of $T^{*} P$ & 13
\end{tabular}

$\begin{array}{lll}5 \text { A cohomology pairing } & 23\end{array}$

$\begin{array}{lll}6 & \text { The holomorphic case } & 29\end{array}$ 
7 Appendix A: The adjoint "representation" of a Lie algebroid

8 Appendix B: The adjoint "representation" and modular class of a Lie groupoid

\section{Introduction}

The notion of Lie algebroids generalizes that of both Lie algebras and tangent bundles. Precisely, a Lie algebroid over a smooth manifold $P$ is a vector bundle $A$ over $P$ together with 1$)$ a Lie algebra structure on the space $\Gamma(A)$ of smooth sections of $A$, and 2) a bundle map $\rho: A \rightarrow T P$, such that

1) $\rho$ defines a Lie algebra homomorphism from $\Gamma(A)$ to the space $\chi^{1}(P)$ of vector fields with the commutator Lie algebra structure, and

2) for $f \in C^{\infty}(P)$ and $\omega_{1}, \omega_{2} \in \Gamma(A)$, the following derivation law holds:

$$
\left\{\omega_{1}, f \omega_{2}\right\}=f\left\{\omega_{1}, \omega_{2}\right\}+\left(\rho\left(\omega_{1}\right) f\right) \omega_{2} .
$$

The map $\rho: A \rightarrow T P$ is called the anchor map of the Lie algebroid $A$. A representation of a Lie algebroid $A$ over $P$ is a vector bundle $E$ over $P$, together with an $\mathbb{R}$-bilinear map (we will deal with the complex case later)

$$
\Gamma(A) \times \Gamma(E) \longrightarrow \Gamma(E): a \otimes s \longmapsto D_{a} s,
$$

where $\Gamma(E)$ denotes the space of smooth sections of $E$, such that for any $a, b \in \Gamma(A), s \in$ $\Gamma(E)$ and $f \in C^{\infty}(P)$,

$$
\begin{aligned}
& \text { (1) } D_{f a} s=f D_{a} s \\
& \text { (2) } D_{a}(f s)=f D_{a} s+(\rho(a) f) s \\
& \text { (3) } D_{a}\left(D_{b} s\right)-D_{b}\left(D_{a} s\right)=D_{[a, b]} s
\end{aligned}
$$

Any Lie algebra is a Lie algebroid over a one point space, and its representations are representations of this Lie algebra. The tangent bundle $T P$ is a Lie algebroid over $P$ with the identity map of $T P$ as the anchor map. Representations of $T P$ are vector bundles over $P$ with flat connections. The trivial representation of a Lie algebroid $A$ is, by definition, the representation of $A$ on the trivial line bundle over $P$ with the action given by

$$
D_{a} f=\rho(a) \cdot f, \quad a \in \Gamma(A), f \in C^{\infty}(P) .
$$


Our motivating example is, however, the cotangent bundle Lie algebroid of a Poisson manifold: if $P$ is a Poisson manifold with the Poisson bivector field $\pi$, the cotangent bundle $T^{*} P$ of $P$ has a natural Lie algebroid structure, where the anchor map $\tilde{\pi}: T^{*} P \rightarrow T P$ is defined by

$$
\tilde{\pi}(p): T_{p}^{*} P \longrightarrow T_{p} P: \alpha_{p} \longmapsto \alpha_{p} \downarrow \pi(p),
$$

and the Lie bracket of 1 -forms $\alpha$ and $\beta$ is given by

$$
\begin{aligned}
\{\alpha, \beta\} & =d \pi(\alpha, \beta)+\tilde{\pi}(\alpha) \downarrow d \beta-\tilde{\pi}(\beta) \downarrow d \alpha \\
& =-d \pi(\alpha, \beta)+L_{\tilde{\pi}(\alpha)} \beta-L_{\tilde{\pi}(\beta)} \alpha .
\end{aligned}
$$

All the basic geometrical aspects of the Poisson structure $\pi$ such as its symplectic leaves and transversal Lie algebra structures to the symplectic leaves are reflected in the Lie algebroid structure on $T^{*} P$.

Other examples of Lie algebroids are the gauge Lie algebroids of principal bundles, the boundary Lie algebroids in $b$-calculus and the Weyl Lie algebroids in Fedosov quantization.

Given a representation $E$ of a Lie algebroid $A$, one can define the Lie algebroid cohomology of $A$ with coefficients in $E$ (see Section 2). When $A$ is a Lie algebra or the tangent bundle $T P$, the Lie algebroid cohomology of $A$ is the Lie algebra cohomology or the de Rham cohomology of $P$. When $A=T^{*} P$ is the cotangent bundle Lie algebroid of a Poisson manifold $P$, the Lie algebroid cohomology of $A$ (with trivial coefficients) is called the Poisson cohomology of $P$.

In this paper, we first construct, for an arbitrary Lie algebroid $A$ over $P$, an intrinsic representation of $A$ on the line bundle $Q_{A}=\wedge^{t o p} A \otimes \wedge^{t o p} T^{*} P$. When $A$ is the subbundle of $T P$ tangent to a foliation $\mathcal{F}$, sections of $Q_{A}$ are the transverse measures to the foliation, and we recover the Bott connection for this foliation. Because of this, for a general Lie algebroid $A$, we may think of sections of $Q_{A}$ as transverse measures to $A$.

We give two applications of the construction of the representation on $Q_{A}$.

The first application concerns the modular class of $A$. In [W], the third author showed that there is a canonical class in the first Poisson cohomology space for each Poisson manifold $P$ called the modular class of $P$, which measures the extent to which the Hamiltonian vector fields on $P$ fail to preserve volume forms on $P$ (when $P$ is orientable). It is the semi-classical 
counterpart of the theory of modular automorphism groups for Von Neumann algebras. By using the Poisson structure on the dual bundle $A^{*}$ of a Lie algebroid $A$, he shows that there is also a canonical class, called the modular class of $A$, in the first Lie algebroid cohomology space of $A$ (with trivial coefficients). We show that this modular class of $A$ can be directly constructed from the representation on $Q_{A}$. We also treat the case of holomorphic Lie algebroids in Section 6. We explain the obstruction for the existence of the modular class in this case in terms of the Chern class of the line bundle $Q_{A}$.

When $A$ is the cotangent bundle Lie algebroid $T^{*} P$ of a Poisson manifold $P$, we have $Q_{A}=\left(\wedge^{t o p} T^{*} P\right)^{2}$, and we show that there is, in fact, a representation of $A=T^{*} P$ on the "square root" $\wedge^{t o p} T^{*} P$ of $Q_{A}$. We also show that the cochain complex that calculates the Lie algebroid cohomology of $T^{*} P$ with coefficients in $\wedge^{t o p} T^{*} P$ is isomorphic to chain complex on differential forms on $P$ introduced by Koszul [K] and studied by Brylinski [Br].

As the second application of the representation of $A$ on $Q_{A}$, we establish a pairing between the Lie algebroid cohomology of $A$ with trivial coefficients and that with coefficients in $Q_{A}$. This generalizes the pairing that gives Poincare duality for Lie algebra cohomology or for de Rham cohomology. As a special case, we get a pairing between Poisson cohomology and what we call the "twisted Poisson cohomology". This pairing may, however, be degenerate in general, as is seen by examples. The problem of when it is non-degenerate is very interesting. We hope to look at it in the future.

\section{Differential calculus on Lie algebroids}

In this section, we list some facts on the calculus on Lie algebroids that will be used in this paper. See Ma for more details.

Let $A$ be a Lie algebroid over $P$ with anchor map $\rho$. For $k \geq 0$, let $\Gamma\left(\wedge^{k} A^{*}\right)$ be the space of smooth sections of $\wedge^{k} A^{*}$. Define

$$
\begin{aligned}
d_{A} & : \Gamma\left(\wedge^{k-1} A^{*}\right) \longrightarrow \Gamma\left(\wedge^{k} A^{*}\right): \\
\left(d_{A} \xi\right)\left(a_{1}, \ldots, a_{k}\right) & =\sum_{i}(-1)^{i+1} \rho\left(a_{i}\right)\left(\xi\left(a_{1}, \ldots, \hat{a}_{i}, \ldots, a_{k}\right)\right) \\
& +\sum_{i<j}(-1)^{i+j} \xi\left(\left\{a_{i}, a_{j}\right\}, \ldots, \hat{a}_{i}, \ldots, \hat{a}_{j}, \ldots, a_{k}\right) .
\end{aligned}
$$


It is well-defined and satisfies $d_{A}^{2}=0$. The cohomology of $\left(\Gamma\left(\wedge^{\bullet} A^{*}\right), d_{A}\right)$ is called the Lie algebroid cohomology of $A$ (with trivial coefficients), and it is denoted by $H^{\bullet}(A)$.

In the case when $A=T P$ is the tangent bundle of $P$, the Lie algebroid cohomology of $A$ is nothing but the de Rham cohomology of $P$.

In the case when $A=T^{*} P$ is the cotangent bundle Lie algebroid of a Poisson manifold $(P, \pi)$, the Lie algebroid cohomology of $A$ is called the Poisson cohomology of $(P, \pi)$.

Suppose that $E$ is a representation of $A$. Let

$$
\Gamma^{k}\left(A^{*}, E\right)=\Gamma\left(\wedge^{k} A^{*}\right) \otimes \Gamma(E)
$$

be the space of " $k$-forms" on $A$ with values in $E$. We regard the action of $A$ on $E$ as defining a map

$$
D: \Gamma^{0}\left(A^{*}, E\right)=\Gamma(E) \longrightarrow \Gamma^{1}\left(A^{*}, E\right) .
$$

Using $d_{A}$, we can extend $D$ to a map

$$
D: \Gamma^{k}\left(A^{*}, E\right) \longrightarrow \Gamma^{k+1}\left(A^{*}, E\right)
$$

by the rule

$$
D(\xi \otimes s)=d_{A} \xi \otimes s+(-1)^{k} \xi \otimes D s,
$$

where $\xi \in \Gamma\left(\wedge^{k} A^{*}\right)$ and $s \in \Gamma(E)$. Then $D^{2}=0$. The cohomology of $\left(\Gamma^{\bullet}\left(A^{*}, E\right), D\right)$ is called the cohomology of $A$ with coefficients in $E$. The operator $D$ satisfies

$$
D(\xi \wedge \eta \otimes s)=d_{A} \xi \wedge \eta \otimes s+(-1)^{|\xi|} \xi \wedge D(\eta \otimes s)
$$

for $\xi, \eta \in \Gamma\left(\wedge^{\bullet} A^{*}\right)$ and $s \in \Gamma(E)$.

The Lie bracket on the sections of $A$ can be extended to the so-called Schouten bracket [ , ] on the space $\Gamma\left(\wedge^{\bullet} A\right)=\oplus_{k} \Gamma\left(\wedge^{k} A\right)$ of multi-sections of $A$. It is characterized by the following properties: for $f, f_{1}, f_{2} \in \Gamma\left(\wedge^{0} A\right)=C^{\infty}(P), a, a_{1}, a_{2} \in \Gamma(A)$ and $X, Y, Z \in$ $\Gamma(\wedge \bullet A)$

$$
\begin{aligned}
& {[X, Y] \in \Gamma\left(\wedge^{|X|+|Y|-1} A\right) ;} \\
& {[a, f]=\rho(a) \cdot f ;} \\
& {\left[a_{1}, a_{2}\right] \text { is the Lie bracket in } \Gamma(A) ;} \\
& {[X, Y]=-(-1)^{(|X|-1)(|Y|-1)}[Y, X] ;} \\
& {[X, Y \wedge Z]=[X, Y] \wedge Z+(-1)^{(|X|-1)|Y|} Y \wedge[X, Z] .}
\end{aligned}
$$


Explicitly, for $\xi \in \Gamma\left(\wedge^{|X|+|Y|-1} A^{*}\right)$, we have,

$$
(\xi,[X, Y])=(-1)^{(|X|-1)(|Y|-1)} i_{X} d_{A} i_{Y} \xi-i_{Y} d_{A} i_{X} \xi+(-1)^{|X|} i_{X \wedge Y} d_{A} \xi,
$$

where $i_{X}$ is the contraction operator by $X$, i.e.,

$$
\left(i_{X} \xi\right)(Y)=(\xi, X \wedge Y)
$$

We now recall the Lie derivative operators on $A$. For a section $a \in \Gamma(A)$, let

$$
L_{a}: \Gamma\left(\wedge^{k} A\right) \longrightarrow \Gamma\left(\wedge^{k} A\right), k \geq 0
$$

be given by

$$
L_{a}\left(a_{1} \wedge a_{2} \wedge \cdots \wedge a_{k}\right)=\left[a, a_{1} \wedge a_{2} \wedge \cdots \wedge a_{k}\right]=\sum_{i=1}^{k} a_{1} \wedge \cdots \wedge\left[a, a_{i}\right] \wedge \cdots \wedge a_{k},
$$

where $a_{i} \in \Gamma(A), i=1,2, \ldots, k$. It is well-defined. We have, for $a, b \in \Gamma(A)$ and $X \in \Gamma(\wedge \bullet A)$,

$$
\begin{aligned}
L_{[a, b]} & =L_{a} L_{b}-L_{b} L_{a} \\
L_{f a} X & \left.=f L_{a} X-a \wedge\left(d_{A} f\right\lrcorner X\right) \\
L_{a}(f X) & =f L_{a} X+(\rho(a) \cdot f) X .
\end{aligned}
$$

We use the same letter $L_{a}$ to denote the operator on $\Gamma\left(\wedge^{\bullet} A^{*}\right)$ given by

$$
\left(L_{a} \xi, X\right)+\left(\xi, L_{a} X\right)=\rho(a) \cdot(\xi, X)
$$

where $\xi \in \Gamma\left(\wedge A^{*}\right)$, and $X \in \Gamma(\wedge A)$. Then

$$
\begin{aligned}
L_{a} & =d_{A} i_{a}+i_{a} d_{A} \\
L_{[a, b]} & =L_{a} L_{b}-L_{b} L_{a} \\
L_{f a} \xi & =f L_{a} \xi+d_{A} f \wedge i_{a} \xi \\
L_{a}(f \xi) & =f L_{a} \xi+(\rho(a) \cdot f) \xi .
\end{aligned}
$$

In particular, if $X \in \Gamma\left(\wedge^{t o p} A\right)$ and $\xi \in \Gamma\left(\wedge^{t o p} A^{*}\right)$, we have, for any $a \in \Gamma(A)$,

$$
\begin{aligned}
L_{f a} X & =f L_{a} X-(\rho(a) \cdot f) X \\
L_{a}(f X) & =f L_{a} X+(\rho(a) \cdot f) X \\
L_{f a} \xi & =f L_{a} \xi+(\rho(a) \cdot f) \xi \\
L_{a}(f \xi) & =f L_{a} \xi+(\rho(a) \cdot f) \xi .
\end{aligned}
$$




\section{The representation of $A$ on $Q_{A}$ and the modular class of $A$}

Assume that a Lie algebroid $A$ acts on a real line bundle $L$ over $P$. We first assume that $L$ has a nowhere vanishing section; thus $L$ is trivial as a line bundle over $P$.

Let $s$ be a nowhere vanishing section of $L$. For a section $a$ of $A$, define $\theta_{s}(a) \in C^{\infty}(P)$ by

$$
D_{a} s=\theta_{s}(a) s
$$

Clearly $\theta_{s} \in \Gamma\left(A^{*}\right)$. Moreover, it follows from $D_{[a, b]} s=D_{a} D_{b} s-D_{b} D_{a} s$ that

$$
\theta_{s}([a, b])=\rho(a) \cdot \theta_{s}(b)-\rho(b) \cdot \theta_{s}(a)
$$

for any two sections $a$ and $b$ of $A$. Thus $\theta_{s}$ is a 1-cocycle with respect to $d_{A}$. If $s_{1}$ is another nowhere vanishing section of $L$, and if $s_{1}=f_{1} s$ for a nowhere vanishing function $f_{1}$, we have

$$
\theta_{s_{1}}=\theta_{s}+d_{A}\left(\log \left|f_{1}\right|\right) \in \Gamma\left(A^{*}\right) .
$$

Thus $\left[\theta_{s_{1}}\right]=\left[\theta_{s}\right] \in H^{1}(A)$. We denote this class in $H^{1}(A)$ by $\theta_{L}$.

Suppose that $L_{1}$ and $L_{2}$ are two line bundle representations of $A$. Equip the tensor product line bundle $L_{1} \otimes L_{2}$ with the representation of $A$ given by

$$
D_{a}\left(s_{1} \otimes s_{2}\right)=D_{a}\left(s_{1}\right) \otimes s_{2}+s_{1} \otimes D_{a}\left(s_{2}\right)
$$

for $a \in \Gamma(A)$. Then, assuming both $L_{1}$ and $L_{2}$ have nowhere vanishing sections, we have $\theta_{L_{1} \otimes L_{2}}=\theta_{L_{1}}+\theta_{L_{2}}$. In particular, $\theta_{L^{2}}=2 \theta_{L}$, where $L^{2}=L \otimes L$.

For a general line bundle $L$ that does not necessarily have a nowhere vanishing section, we define

$$
\theta_{L}=\frac{1}{2} \theta_{L^{2}} \in H^{1}(A)
$$

Since the square of any real line bundle over $P$ is trivial as a line bundle, the class $\theta_{L^{2}}$ is defined. By the remark above, $\theta_{L}$ is well-defined.

Definition 3.1 The class $\theta_{L} \in H^{1}(A)$ is called the characteristic class of $A$ associated to the representation $L$.

The following Proposition is immediate. 
Proposition 3.2 For any two line bundles $L_{1}$ and $L_{2}$ with $A$ representations,

$$
\theta_{L_{1} \otimes L_{2}}=\theta_{L_{1}}+\theta_{L_{2}}
$$

Our notion of characteristic class is very much like that for flat line bundles.

Example 3.3 The characteristic class of $A$ associated to the trivial representation is zero.

Example 3.4 Any Lie algebra homomorphism

$$
\rho: \mathfrak{g} \longrightarrow \chi^{1}(P)
$$

from a Lie algebra $\mathfrak{g}$ to the Lie algebra $\chi^{1}(P)$ of vector fields on $P$ defines a Lie algebroid structure on the trivial vector bundle $A=P \times \mathfrak{g}$ called the transformation Lie algebroid. The anchor map is $\rho$, now regarded as a bundle map from $P \times \mathfrak{g}$ to $T P$. The Lie bracket on the space $\Gamma(P \times \mathfrak{g}) \cong C^{\infty}(P, \mathfrak{g})$ of smooth sections of $P \times \mathfrak{g}$ is given by

$$
\{\bar{x}, \bar{y}\}=[\bar{x}, \bar{y}]_{\mathfrak{g}}+\rho_{\bar{x}} \cdot \bar{y}-\rho_{\bar{y}} \cdot \bar{x},
$$

where the first term on the right hand side denotes the pointwise Lie bracket in $\mathfrak{g}$, and the second term denotes the derivative of the $\mathfrak{g}$-valued function $\bar{y}$ in the direction of the vector field $\rho_{\bar{x}}$.

Suppose that $U$ is a vector space on which the Lie algebra $\mathfrak{g}$ acts. Then there is a representation of $A$ on the trivial vector bundle $P \times U$ given by

$$
D_{\bar{x}} \bar{u}=\rho_{\bar{x}} \cdot \bar{u}+\bar{x}(\bar{u})
$$

where $\bar{u} \in C^{\infty}(P, U)$ is a section of $P \times U, \rho_{\bar{x}} \cdot \bar{u}$ is the pointwise derivative of $\bar{u}$ in the direction of $\rho_{\bar{x}}$, and $\bar{x}(\bar{u})$ denotes the action of $\bar{x} \in C^{\infty}(P, \mathfrak{g})$ on $\bar{u} \in C^{\infty}(P, U)$ taken pointwise over $P$. When $U$ is finite dimensional, we get a representation of $A=P \times \mathfrak{g}$ on the trivial line bundle $P \times\left(\wedge^{t o p} U\right)$. Let $\chi_{U} \in \mathfrak{g}^{*}$ be the character of $\mathfrak{g}$ associated to $U$. Then the characteristic class of the transformation Lie algebroid $A=P \times \mathfrak{g}$ associated to the line bundle $P \times\left(\wedge^{t o p} U\right)$ is given by the constant section of $A^{*}=P \times \mathfrak{g}^{*}$ defined by $\chi_{U}$. In particular, there is a representation of $A$ on the line bundle $\wedge^{t o p} A=P \times\left(\wedge^{t o p} \mathfrak{g}\right)$ coming from the adjoint action of $\mathfrak{g}$ on $\wedge^{\text {top }} \mathfrak{g}$, and the corresponding characteristic class of $A$ is given by the constant section of $A^{*}$ defined by the adjoint character $\xi_{0} \in \mathfrak{g}^{*}$ of $\mathfrak{g}$. 
There is also a natural representation of the transformation Lie algebroid $A=P \times \mathfrak{g}$ on the tangent bundle $T P$ of $P$ via

$$
D_{\bar{x}} V=\left[\rho_{\bar{x}}, V\right]+\rho_{V \cdot \bar{x}}
$$

where $V$ is a vector field on $P$ and $V \cdot \bar{x}$ denotes the Lie derivative of $\bar{x}$ in the direction of $V$. Correspondingly, there is a representation of $P \times \mathfrak{g}$ on the cotangent bundle $T^{*} P$ satisfying

$$
\rho_{\bar{x}}(\alpha, V)=\left(D_{\bar{x}} \alpha, V\right)+\left(\alpha, D_{\bar{x}} V\right)
$$

for any 1-form $\alpha$ on $P$. Equivalently,

$$
\left(D_{\bar{x}} \alpha, V\right)=\rho_{\bar{x}}(\alpha, V)-\left(\alpha,\left[\rho_{\bar{x}}, V\right]+\rho_{V \cdot \bar{x}}\right)=\left(L_{\rho_{\bar{x}}} \alpha\right)(V)-\left(\alpha, \rho_{V \cdot \bar{x}}\right)
$$

Consequently, there is a representation of $A$ on the line bundle $\wedge^{t o p} T^{*} P$, as well as one on the density bundle $\left|\wedge^{\text {top }} T^{*} P\right|$ of $P$ (see $[\overline{\mathrm{B}-\mathrm{T}}]$ ). Let $\mu$ be a non-vanishing density on $P$. Recall that the divergence of a vector field $V$ on $P$ with respect to $\mu$ is defined to be the function $\operatorname{div}_{\mu} V$ given by

$$
L_{V} \mu=\left(\operatorname{div}_{\mu} V\right) \mu .
$$

The characteristic class of $A$ associated to $\wedge^{\text {top }} T^{*} P$ or the density bundle $\left|\wedge^{\text {top }} T^{*} P\right|$ is now given by the section of $A^{*}$ that associates to each constant section $x$ of $A$ corresponding to $x \in \mathfrak{g}$ the function $\operatorname{div}_{\mu} \rho_{x}$.

We now show that every Lie algebroid has an intrinsic line bundle representation.

Let $A$ be a Lie algebroid over $P$ with anchor map $\rho$. Consider the line bundle

$$
Q_{A}=\wedge^{t o p} A \otimes \wedge^{t o p} T^{*} P
$$

For a section $a$ of $A$, define

$$
D_{a}: \Gamma\left(Q_{A}\right) \longrightarrow \Gamma\left(Q_{A}\right)
$$

by

$$
D_{a}(X \otimes \mu)=L_{a}(X) \otimes \mu+X \otimes L_{\rho(a)} \mu,
$$

where $X \in \Gamma\left(\wedge^{t o p} A\right), \mu \in \Gamma\left(\wedge^{t o p} T^{*} P\right)$, and $L_{\rho(a)} \mu$ denotes the Lie derivative of $\mu$ in the direction of $\rho(a)$. It follows from (21) and (23) that $D_{a}$ is well-defined. 
Theorem 3.5 The map

$$
\Gamma(A) \otimes \Gamma\left(Q_{A}\right) \longrightarrow \Gamma\left(Q_{A}\right): a \otimes(X \otimes \mu) \longmapsto D_{a}(X \otimes \mu)
$$

given by (31) defines a representation of $A$ on the line bundle $Q_{A}$.

Proof. The proof is straightforward. The underlying idea is given in Remark 3.7.

\section{Q.E.D.}

Definition 3.6 We define the modular class of $A$ to be the characteristic class associated to the representation of $A$ on the line bundle $Q_{A}$, and we denote it by $\theta_{A}$

Remark 3.7 Alternatively, we can define $Q_{A}$ as $H o m\left(\wedge^{t o p} A^{*}, \wedge^{t o p} T^{*} P\right)$. There is a Lie derivative action of $A$ on $\wedge^{t o p}\left(A^{*}\right)$, and a Lie derivative action of $A$ on $\wedge^{t o p}\left(T^{*} P\right)$ defined using the anchor map and the usual Lie derivative of vector fields. Neither of these Lie derivative actions defines a representation of $A$, but we can combine these two Lie derivatives to get a representation of $A$ on $Q_{A}$ by the formula

$$
\left(D_{a} \phi\right)(\xi)=L_{\rho(a)}(\phi(\xi))-\phi\left(L_{a} \xi\right)
$$

When we calculate $\left(D_{f a} \phi\right)(\xi)$, each Lie derivative contributes a term $(\rho(a) f) \phi(\xi)$, and these two terms cancel:

$$
\begin{aligned}
\left(D_{f a} \phi\right)(\xi) & =f \cdot L_{\rho(a)}(\phi(\xi))+(\rho(a) f) \phi(\xi)-f \phi\left(L_{a} \xi\right)-(\rho(a) f) \phi(\xi) \\
& =f D_{a} \phi(\xi) .
\end{aligned}
$$

Thus we obtain $D_{f a}=f D_{a}$. In this interpretation, the representation of $A$ on $Q_{A}$ is the difference between the Lie derivative action of $a$ on $\wedge^{t o p} A^{*}$ and the Lie derivative action on $\wedge^{\text {top }} T^{*} P$ defined via the anchor map.

This construction can also be interpreted as follows: the relations of the Lie derivative imply that we can give $\wedge^{t o p} A^{*}$ and $\wedge^{t o p} T^{*} P$ the structure of right representations of $A$. Explicitly, we set $r(a) \cdot s=-L_{a}(s)$ and $r(f) \cdot s=f s$, where $f \in C^{\infty}(P)$ and $s$ is a section of $\wedge^{t o p} A^{*}$ and use the analogous construction for $\wedge^{t o p} T^{*} P$. A Lie algebroid has a functorially defined universal enveloping $D$-algebra, which is the associative algebra generated by functions on $P$ and sections of the Lie algebroid subject to the relations induced by the Lie 
algebroid relations (see [B-B]). When we say a module is a right representation of $A$, we mean that it is a right module for the universal enveloping $D$-algebra.

Let $D_{P}$ be the algebra of smooth differential operators on $P$. It is the universal enveloping $D$-algebra for the tangent Lie algebroid $T P$. There is a standard construction in the theory of $D_{P}$-modules for changing a right $D_{P}$-module into a left one. It proceeds by observing that the canonical bundle $\wedge^{t o p} T^{*} P$ is a right $D_{P}$-module and that for any right $D_{P}$-module $M$, there is a new left $D_{P}$-module $\operatorname{Hom}_{C}{ }_{(P)}\left(\wedge^{t o p} T^{*} P, M\right)$ with action given by the formula:

$$
(a \cdot \phi)(\omega)=a(\phi(\omega))-\phi\left(L_{a} \omega\right)
$$

where $a$ is a vector field on $P$ (see [B] $)$. The action of $C^{\infty}(P)$ is by multiplication on $M$ and this makes $H_{o m} C_{(P)}\left(\wedge^{t o p} T^{*} P, M\right)$ into a left $D_{P}$-module. Our construction of the representation of $A$ on $Q_{A}$ is just the Lie algebroid analog of this construction applied to the right Lie algebroid module $\wedge^{t o p} T^{*} P$. It would be of interest to apply more serious ideas from $D$-module theory to Lie algebroids. We hope to return to this subject in the future.

Remark 3.8 Another definition of the modular class begins with the observation of Kontsevich $\mathrm{K}$ ] that a Lie algebroid $A$ can be viewed as a supermanifold $A_{s}$, with $\Gamma\left(\wedge^{\bullet} A^{*}\right)$ as its algebra of functions. The differential $d_{A}$ defining Lie algebroid cohomology can be considered as a vector field on $A_{s}$. On the other hand, a section $s_{0}$ of $Q_{A}$ determines in a natural way a volume element on $A_{s}$. The divergence of $d_{A}$ with respect to this volume element is a function on $A_{s}$ which is in fact the section $\theta_{s_{0}}$ of $A^{*}$ representing the modular class.

Remark 3.9 When $P$ is not orientable, we can also replace $\wedge^{t o p} T^{*} P$ by the density bundle $\left|\wedge^{\text {top }} T^{*} P\right|$, i.e., define

$$
Q_{A}=\wedge^{t o p} A \otimes\left|\wedge^{t o p} T^{*} P\right|
$$

Then formula (31) still defines a representation of $A$ on $Q_{A}$.

Example 3.10 For $A=T P$ and when $P$ is orientable, we have

$$
Q_{A}=\wedge^{t o p} T P \otimes \wedge^{t o p} T^{*} P \cong H o m\left(\wedge^{t o p} T^{*} P, \wedge^{t o p} T^{*} P\right) .
$$


It has a canonical nowhere vanishing section given by the identity morphism. It is clear from (32) that

$$
D_{V} s=0
$$

for every vector field $V$ on $P$. Thus the modular class of $T P$ is zero. When $P$ is not orientable, let $\mathcal{O}$ be the orientation bundle for $P$. Then $\left|\wedge^{t o p} T^{*} P\right|=\wedge^{t o p} T^{*} P \otimes \mathcal{O}$, and thus $Q_{A} \cong \mathcal{O}$.

Because of Example 3.10, we can view $Q_{A}$ for a Lie algebroid $A$ as the orientation bundle of $A$.

Example 3.11 When $A$ is an integrable subbundle of $T P$, the line bundle $Q_{A}$ is isomorphic to the top exterior power of the conormal bundle to the corresponding foliation $\mathcal{F}$ (assuming the normal bundle is orientable - otherwise the twisting by the orientation bundle is needed), and thus sections of $Q_{A}$ are transverse measures to $\mathcal{F}$. The representation of $A$ on $Q_{A}$ is nothing but the Bott connection of the foliation. It is thus appropriate to call the modular class of $A$ the modular class of the foliation.

Because of Exam 3.11, for a general Lie algebroid $A$, we should think of sections of $Q_{A}$ as being "transverse measures to $A$ ".

Example 3.12 When a Lie algebra $\mathfrak{g}$ is considered as a Lie algebroid over a one point space, its modular class is given by the adjoint character $\xi_{0} \in \mathfrak{g}^{*}$ :

$$
\left(\xi_{0}, x\right)=\operatorname{tr}\left(a d_{x}\right), x \in \mathfrak{g} .
$$

Example 3.13 Let $A=P \times \mathfrak{g}$ be the transformation Lie algebroid (see Example 3.4). In this case, there is a representation of $A$ on each of the line bundles $\wedge^{t o p} A$ and $\wedge^{t o p} T^{*} P$. The representation of $A$ on $Q_{A}$ is simply the tensor product of these two. Let $\mu$ be a nowhere vanishing density on $P$. Then the modular class of $A$ is given by the section of $A^{*}$ that assigns to each section $\bar{x}$ of $A$ the function $\left(\xi_{0}, \bar{x}\right)-\operatorname{div}_{\mu}\left(\rho_{\bar{x}}\right)$, where $\xi_{0} \in \mathfrak{g}^{*}$ is the adjoint character of $\mathfrak{g}$. 


\section{The case of $T^{*} P$}

In this section, we treat the case of the cotangent bundle Lie algebroid $T^{*} P$ of a Poisson manifold $P$. We will show that the modular class we define here is twice the modular class of $P$ as a Poisson manifold defined in $\mathrm{We}$. We first recall that definition.

Consider the density bundle $\left|\wedge^{t o p} T^{*} P\right|$ of $P$. Let $\mu$ be a nowhere vanishing density on $P$. For a function $f$ on $P$, define $w_{\mu}(f) \in C^{\infty}(P)$ by

$$
L_{\tilde{\pi}(d f)} \mu=w_{\mu}(f) \mu .
$$

One then checks that $w_{\mu}(f)$ is a derivation with respect to $f$, so it is given by a vector field, which we denote by $w_{\mu}$. It also satisfies, for any 1 -forms $\alpha$ and $\beta$ on $P$,

$$
w_{\mu}(\{\alpha, \beta\})=\tilde{\pi}(\alpha) \cdot w_{\mu}(\beta)-\tilde{\pi}(\beta) \cdot w_{\mu}(\alpha) .
$$

(This is equivalent to $L_{w_{\mu}} \pi=0$.) Thus, $w_{\mu}$ defines a class in the first Poisson cohomology $H_{\pi}^{1}(P)$ of $P$. If $\mu_{1}=f_{1} \mu$ is another nowhere vanishing density on $P$, the two vector fields $w_{\mu}$ and $w_{\mu_{1}}$ are related by

$$
w_{\mu_{1}}=w_{\mu}-\tilde{\pi}\left(d \log \left|f_{1}\right|\right) \text {. }
$$

Thus $w_{\mu}$ and $w_{\mu_{1}}$ define the same cohomology class in $H_{\pi}^{1}(P)$. It is called the modular class of $(P, \pi)$ in $\mathrm{We}$.

We now look at the modular class $\theta_{T^{*} P}$ given by Definition 3.6. The line bundle $Q_{A}$ in this case is

$$
Q_{A}=\left(\wedge^{t o p} T^{*} P\right)^{2}
$$

The representation of $T^{*} P$ on $\left(\wedge^{t o p} T^{*} P\right)^{2}$ is given by

$$
D_{\alpha}\left(\mu_{1} \otimes \mu_{2}\right)=\left\{\alpha, \mu_{1}\right\} \otimes \mu_{2}+\mu_{1} \otimes L_{\tilde{\pi}(\alpha)} \mu_{2},
$$

where $\alpha$ is a 1 -form on $P, \mu_{1}$ and $\mu_{2}$ are two top degree forms on $P$, and $\left\{\alpha, \mu_{1}\right\}$ is the Schouten bracket between $\alpha$ and $\mu_{1}$.

We consider the special case when $\alpha=d f$ is an exact 1 -form.

Lemma 4.1 For any differential $k$-form $\xi$ on $P$,

$$
\{d f, \xi\}=L_{\tilde{\pi}(d f)} \xi
$$


Proof. Since the operators $L_{\tilde{\pi}(d f)}$ and $L_{d f}: \xi \mapsto\{d f, \xi\}$ have the same derivation properties with respect to the wedge product on differential forms, it is enough to check the cases when $k=0$ and when $k=1$. The case $k=0$ is obvious from the definition. Now let $\xi$ be a 1-form. From Formula (11), we know that for any 1-form $\alpha$,

$$
\begin{aligned}
\{\alpha, \xi\} & \left.=\left(d i_{\tilde{\pi}(\alpha)}+i_{\tilde{\pi}(\alpha)} d\right) \xi-\tilde{\pi}(\xi)\right\lrcorner d \alpha \\
& \left.=L_{\tilde{\pi}(\alpha)} \xi-\tilde{\pi}(\xi)\right\lrcorner d \alpha .
\end{aligned}
$$

In particular, when $d \alpha=0$, we have

$$
\{\alpha, \xi\}=L_{\tilde{\pi}(\alpha)} \xi
$$

Thus $\{d f, \xi\}=L_{\tilde{\pi}(d f)} \xi$.

\section{Q.E.D.}

It now follows from the Lemma that

$$
D_{d f}\left(\mu_{1} \otimes \mu_{2}\right)=\left(L_{\tilde{\pi}(d f)} \mu_{1}\right) \otimes \mu_{2}+\mu_{1} \otimes\left(L_{\tilde{\pi}(d f)} \mu_{2}\right)
$$

for any two top degree forms $\mu_{1}$ and $\mu_{2}$.

The density bundle $S_{P}$ also satisfies $S_{P}^{2}=\left(\wedge^{t o p} T^{*} P\right)^{2}$. Let $\mu$ be a nowhere vanishing section of $S_{P}$. Then $\mu \otimes \mu=\mu^{2}$ is a nowhere vanishing section of $\left(\wedge^{\text {top }} T^{*} P\right)^{2}$. For any $\alpha=d f$, we have

$$
D_{d f}\left(\mu^{2}\right)=2\left(L_{\tilde{\pi}(d f)} \mu\right) \otimes \mu .
$$

Since $L_{\tilde{\pi}(d f)} \mu=w_{\mu}(f) \mu$, we have

$$
D_{d f}\left(\mu^{2}\right)=2 w_{\mu}(f) \mu^{2}
$$

Thus (recall the definition of $\theta_{\mu^{2}}$ from Section 3) we have

$$
\theta_{\mu^{2}}=2 w_{\mu}
$$

This shows that the modular class $\theta_{T^{*} P}$ of the Lie algebroid $T^{*} P$ we define here is twice the modular class of $P$ as a Poisson manifold defined in $\mathrm{We}$.

Definition 4.2 The modular class $\theta_{P}$ of a Poisson manifold $P$ is defined to be $\theta_{P}=\frac{1}{2} \theta_{T^{*} P}$, i.e., one half of the modular class of the cotangent Lie algebroid $T^{*} P$. 
Thus our definition for $\theta_{P}$ is the same as that in [We].

We now show that there is, in fact, a representation of $T^{*} P$ on the line bundle $\wedge^{t o p} T^{*} P$. We first state the following Proposition. The proof is straightforward, and we omit it.

Proposition 4.3 Assume that $M$ is a line bundle over $P$ such that a Lie algebroid $A$ with anchor map $\rho: A \rightarrow T P$ acts on $M \otimes M=M^{2}$. Then there is a representation of $A$ on $M$ as well that is defined as follows: let a be a section of $A$, and let $s$ be a section of $M$. Let $U$ be an open subset of $P$ over which $t$ is a nowhere vanishing section of $M$. Write $s=f t$ for some function $f$ defined on $U$. Define

$$
\left.\left(D_{a} s\right)\right|_{U}=(\rho(a) \cdot f) t+\frac{1}{2} \frac{D_{a}\left(t^{2}\right)}{t^{2}} s .
$$

This is well-defined, and it defines a representation of $A$ on $M$, whose square gives the original representation of $A$ on $M^{2}$.

We now give the explicit representation of the cotangent bundle Lie algebroid $T^{*} P$ on the line bundle $\wedge^{t o p} T^{*} P$, whose existence is guaranteed by Proposition 4.3. Formula (36) has also been independently discovered by Ping $\mathrm{Xu}$ in $\mathrm{Xu}]$.

Theorem 4.4 Let $P$ be a Poisson manifold with the Poisson bivector field $\pi$. For a 1-form $\alpha$ and a top degree form $\mu$ on $P$, define

$$
D_{\alpha} \mu=\{\alpha, \mu\}-(\pi, d \alpha) \mu
$$

where $\{\alpha, \mu\}$ denotes the Schouten bracket between $\alpha$ and $\mu$, and $(\pi, d \alpha)$ denotes the pairing between $\pi$ and $d \alpha$. Then (34) defines a representation of $T^{*} P$ on $\wedge^{\text {top }} T^{*} P$. We also have

$$
\begin{aligned}
D_{\alpha} \mu & =L_{\tilde{\pi}(\alpha)} \mu+(\pi, d \alpha) \mu \\
& =\alpha \wedge d i_{\pi} \mu .
\end{aligned}
$$


Proof. For any $f \in C^{\infty}(P)$, we have

$$
\begin{aligned}
D_{f \alpha} \mu & =\{f \alpha, \mu\}-(\pi, d(f \alpha)) \mu \\
& =f\{\alpha, \mu\}-(\tilde{\pi}(\alpha) \cdot f) \mu-f(\pi, d \alpha) \mu-(\pi, d f \wedge \alpha) \mu \\
& =f D_{\alpha} \mu-((\pi, \alpha \wedge d f)+(\pi, d f \wedge \alpha)) \mu \\
& =f D_{\alpha} \mu ; \\
D_{\alpha}(f \mu) & =\{\alpha, f \mu\}-(\pi, d \alpha) f \mu \\
& =(\tilde{\pi}(\alpha) \cdot f) \mu+f D_{\alpha} \mu .
\end{aligned}
$$

If $\beta$ is another 1 -form on $P$, we have

$$
\begin{aligned}
D_{\alpha} D_{\beta} \mu-D_{\beta} D_{\alpha} \mu= & D_{\alpha}(\{\beta, \mu\}-(\pi, d \beta) \mu)-D_{\beta}(\{\alpha, \mu\}-(\pi, d \alpha) \mu) \\
= & \{\alpha,\{\beta, \mu\}\}-(\pi, d \alpha)\{\beta, \mu\} \\
& -\tilde{\pi}(\alpha) \cdot(\pi, d \beta) \mu-(\pi, d \beta)\{\alpha, \mu\}+(\pi, d \beta)(\pi, d \alpha) \mu \\
& -\{\beta,\{\alpha, \mu\}\}+(\pi, d \beta)\{\alpha, \mu\} \\
& +\tilde{\pi}(\beta) \cdot(\pi, d \alpha) \mu+(\pi, d \alpha)\{\beta, \mu\}-(\pi, d \alpha)(\pi, d \beta) \mu \\
= & \{\{\alpha, \beta\}, \mu\}-\tilde{\pi}(\alpha) \cdot(\pi, d \beta) \mu+\tilde{\pi}(\beta) \cdot(\pi, d \alpha) \mu \\
= & \{\{\alpha, \beta\}, \mu\}-(\pi, d\{\alpha, \beta\}) \mu \\
= & D_{\{\alpha, \beta\}} \mu .
\end{aligned}
$$

In the last step, we used the fact that

$$
\tilde{\pi}(\alpha) \cdot(\pi, d \beta)-\tilde{\pi}(\beta) \cdot(\pi, d \alpha)=(\pi, d\{\alpha, \beta\}) .
$$

Indeed,

$$
\begin{aligned}
\text { l.h.s } & =([\tilde{\pi}(\alpha), \pi], d \beta)+\left(\pi, L_{\tilde{\pi}(\alpha)} d \beta\right)-([\tilde{\pi}(\beta), \pi], d \alpha)-\left(\pi, L_{\tilde{\pi}(\beta)} d \alpha\right) \\
& =-([\pi, \tilde{\pi}(\alpha)], d \beta)+([\pi, \tilde{\pi}(\beta)], d \alpha)+(\pi, d\{\alpha, \beta\}) \\
& =-(\tilde{\pi}(d \alpha), d \beta)+(\tilde{\pi}(d \beta), d \alpha)+(\pi, d\{\alpha, \beta\}) \\
& =(\pi, d\{\alpha, \beta\}) \\
& =\text { r.h.s. }
\end{aligned}
$$

Here we used Formula (2) and the fact that

$$
\tilde{\pi}: \Gamma\left(\wedge^{\bullet} T^{*} P\right) \longrightarrow \Gamma\left(\wedge^{\bullet} T P\right)
$$


intertwines the operators $d$ and $d_{\pi}=[\pi, \bullet]$.

To show that $D_{\alpha} \mu$ is also given by (35), we first observe that the right hand side of (35) is $C^{\infty}(P)$-linear in $\alpha$. We also know from Lemma 4.1 that (35) holds when $\alpha$ is exact. Thus it holds for an arbitrary $\alpha$. To show (36), we start with

$$
\alpha \wedge i_{\pi} \mu=-i_{\tilde{\pi}(\alpha)} \mu
$$

and apply $d$ to both sides. We get

$$
d \alpha \wedge i_{\pi} \mu-\alpha \wedge d i_{\pi} \mu=-d i_{\tilde{\pi}(\alpha)} \mu
$$

or,

$$
(\pi, d \alpha) \mu-\alpha \wedge d i_{\pi} \mu=-L_{\tilde{\pi}(\alpha)} \mu .
$$

Formula (36) now follows from (35).

\section{Q.E.D.}

The characteristic class of the Lie algebroid $T^{*} P$ associated to this representation on $\wedge^{t o p} T^{*} P$ is now exactly the same as the modular class of $P$ as a Poisson manifold defined in $\mathrm{We}$.

We now consider the Lie algebroid cohomology $H^{\bullet}\left(P, \wedge^{t o p} T^{*} P\right)$ of $T^{*} P$ with coefficients in $\wedge^{t o p} T^{*} P$. We will show that it is isomorphic to the Poisson homology space $H_{\bullet}(P)$ of Koszul [Kz] and Brylinski [B]].

Recall (Section 2) that the complex that gives rise to the Lie algebroid cohomology $H^{\bullet}\left(P, \wedge^{t o p} T^{*} P\right)$ is the space

$$
C_{1}=\chi^{\bullet}(P) \otimes \Gamma\left(\wedge^{t o p} T^{*} P\right)
$$

together with the operator

$$
\delta_{\pi}^{\prime}: \chi^{k}(P) \otimes \Gamma\left(\wedge^{t o p} T^{*} P\right) \longrightarrow \chi^{k+1}(P) \otimes \Gamma\left(\wedge^{t o p} T^{*} P\right)
$$

given by

$$
\delta_{\pi}^{\prime}: V \otimes \mu \longmapsto[\pi, V] \otimes \mu+(-1)^{k} V \wedge D \mu,
$$

where, for $\mu \in \Gamma\left(\wedge^{t o p} T^{*} P\right)$,

$$
D \mu \in \chi^{1}(P) \otimes \Gamma\left(\wedge^{t o p} T^{*} P\right)
$$


is given by

$$
(D \mu)(\alpha)=D_{\alpha} \mu=\{\alpha, \mu\}-(\pi, d \alpha) \mu=L_{\tilde{\pi} \alpha} \mu+(\pi, d \alpha) \mu=\alpha \wedge d i_{\pi} \mu
$$

for any 1-form $\alpha$ on $P$ (see Theorem 4.4).

In $[\mathrm{Kz}]$, Koszul (also see $[\mathrm{Br}]$ ) introduced the operator

$$
\partial_{\pi}=i_{\pi} d-d i_{\pi}: \Omega^{k+1}(P) \longrightarrow \Omega^{k}(P) .
$$

It satisfies $\left(\partial_{\pi}\right)^{2}=0$. The homology of $\left(\Omega^{\bullet}(P), \partial_{\pi}\right)$ is called the Poisson homology of $(P, \pi)$ and it is denoted by $H_{\bullet}(P)$.

Let $n=\operatorname{dim} P$, and for each $k=0,1, \ldots, n$, define

$$
\left.\tau: \chi^{k}(P) \otimes \Gamma\left(\wedge^{n} T^{*} P\right) \longrightarrow \Omega^{n-k}(P): V \otimes \mu \longmapsto i_{V} \mu=V\right\lrcorner \mu .
$$

It is clearly a vector space isomorphism from $C_{1}$ to $\Omega^{\bullet}(P)$.

Theorem 4.5 For any $V \otimes \mu \in C_{1}^{k}=\chi^{k}(P) \otimes \Gamma\left(\wedge^{n} T^{*} P\right)$,

$$
\tau \delta_{\pi}^{\prime}(V \otimes \mu)=(-1)^{k+1} \partial_{\pi} \tau(V \otimes \mu)
$$

Consequently,

$$
H^{k}\left(P, \wedge^{t o p} T^{*} P\right) \cong H_{n-k}(P) .
$$

Proof. Since all operators in (40) are local, we only need to prove that it holds locally. Thus, without loss of generality, we can assume that $P$ is orientable. Let $\mu_{0}$ be a volume form of $P$. We can then identify $\chi^{\bullet}(P)$ with $C_{1}$ by

$$
\chi^{\bullet}(P) \longrightarrow C_{1}: V \longmapsto V \otimes \mu_{0} .
$$

The isomorphism $\tau: C_{1} \rightarrow \Omega^{\bullet}(P)$ now becomes

$$
\left.\tau_{\mu_{0}}: \chi^{k}(P) \longrightarrow \Omega^{n-k}(P): V \longmapsto V\right\lrcorner \mu_{0}=i_{V} \mu_{0}
$$

Let $\theta_{0}$ be the modular vector field associated to $\wedge^{t o p} T^{*} P$ defined by $\mu_{0}$, i.e., for any 1-form $\alpha$,

$$
\theta_{0}(\alpha) \mu_{0}=D_{\alpha} \mu_{0}=L_{\tilde{\pi}(\alpha)} \mu_{0}+(\pi, d \alpha) \mu_{0}
$$


(see Theorem 4.4). Then the operator $\delta_{\pi}^{\prime}$ becomes

$$
\delta_{\pi, \mu_{0}}^{\prime}: \chi^{k}(P) \longrightarrow \chi^{k+1}(P): V \longmapsto[\pi, V]+\theta_{0} \wedge V
$$

and (40) becomes

$$
\left.\left.\left.\left.\left([\pi, V]+\theta_{0} \wedge V\right)\right\lrcorner \mu_{0}=(-1)^{k+1}(\pi\lrcorner d(V\lrcorner \mu_{0}\right)-d((V \wedge \pi)\lrcorner \mu_{0}\right)\right)
$$

for $V \in \chi^{k}(P)$.

Introduce the operator

$$
\left.\left.b_{\mu_{0}}: \chi^{k}(P) \longrightarrow \chi^{k-1}(P):\left(b_{\mu_{0}} V\right)\right\lrcorner \mu_{0}=(-1)^{k} d(V\lrcorner \mu_{0}\right) .
$$

Clearly $b_{\mu_{0}}^{2}=0$.

Lemma 4.6 For any $V_{1}, V_{2} \in \chi^{\bullet}(P)$,

$$
b_{\mu_{0}}\left(V_{1} \wedge V_{2}\right)=b_{\mu_{0}}\left(V_{1}\right) \wedge V_{2}+(-1)^{\left|V_{1}\right|} V_{1} \wedge b_{\mu_{0}}\left(V_{2}\right)+(-1)^{\left|V_{1}\right|}\left[V_{1}, V_{2}\right] ;
$$

proof. It follows from Formula (10) for the Schouten bracket $\left[V_{1}, V_{2}\right]$ that the two sides of (46) are equal when paired with any for $\alpha$ of degree $\left|V_{1}\right|+\left|V_{2}\right|-1$.

\section{Q.E.D.}

\section{Lemma 4.7}

$$
b_{\mu_{0}} \pi=\theta_{0}
$$

Proof. This is an immediate consequence of $(36)$.

\section{Q.E.D.}

We now continue with the proof of Theorem 4.5. We need to prove (44). Using again the operator $b_{\mu_{0}}$ and its property (46), we see that

$$
\begin{aligned}
\text { r.h.s. of (44) } & \left.=\left(b_{\mu_{0}}(V \wedge \pi)-b_{\mu_{0}} V \wedge \pi\right)\right\lrcorner \mu_{0} \\
& \left.=\left((-1)^{k} V \wedge b_{\mu_{0}} \pi+(-1)^{k}[V, \pi]\right)\right\lrcorner \mu_{0} \\
& \left.=\left(b_{\mu_{0}} \pi \wedge V[\pi, V]\right)\right\lrcorner \mu_{0} \\
& \left.=\left(\theta_{0} \wedge V+[\pi, V]\right)\right\lrcorner \mu_{0} \\
& =\text { l.h.s. of (44). }
\end{aligned}
$$

This completes the proof of Theorem 4.5. 
Q.E.D.

We now look at the case when $G$ is a Poisson Lie group. Let $(G, \pi)$ be a connected Poisson Lie group with tangent Lie bialgebra $\left(\mathfrak{g}, \mathfrak{g}^{*}\right)$ (see, for example, [STS] and [L-W]). Let $\xi_{0} \in \mathfrak{g}$ and $x_{0} \in \mathfrak{g}$ be respectively the characters of the Lie algebras $\mathfrak{g}$ and $\mathfrak{g}^{*}$ with respect to their adjoint representations, i.e., for any $x \in \mathfrak{g}$ and $\xi \in \mathfrak{g}^{*}$,

$$
\begin{aligned}
& \left(\xi_{0}, x\right)=\operatorname{tr}\left(a d_{x}\right) \\
& \left(x_{0}, \xi\right)=\operatorname{tr}\left(a d_{\xi}\right) .
\end{aligned}
$$

Denote respectively by $x_{0}^{l}$ and $x_{0}^{r}$ the left and right invariant vector fields on $G$ whose values at the identity element $e$ are $x_{0}$. Denote by $\xi_{0}^{r}$ the right invariant 1 -form on $G$ whose value at $e$ is $\xi_{0}$. Recall that the right dressing vector field [L-W] [STS defined by $\xi_{0}$ is the vector field

$$
\sigma_{\xi_{0}}=\tilde{\pi}\left(\xi_{0}^{r}\right)
$$

Proposition 4.8 Let $\mu$ be a right invariant volume form on $G$. The vector field $\theta_{\mu}$ on $G$ defined by $\mu$ :

$$
\left(\theta_{\mu}, \alpha\right):=\frac{D_{\alpha} \mu}{\mu}
$$

is given by

$$
\theta_{\mu}=\frac{1}{2}\left(x_{0}^{l}+x_{0}^{r}-\sigma_{\xi_{0}}\right) .
$$

Similarly, if $\mu^{l}$ is a left invariant volume form on $G$. The vector field $\theta_{\mu^{l}}$ on $G$ is given by

$$
\theta_{\mu^{l}}=\frac{1}{2}\left(x_{0}^{l}+x_{0}^{r}+\sigma_{\xi_{0}}\right) .
$$

Proof. Let $\alpha$ be a right invariant 1 -form on $G$. The Schouten bracket $\{\alpha, \mu\}$ is also right invariant, and is equal to $\left(x_{0}^{r}, \alpha\right) \mu$. Consider the vector field $v$ on $G$ defined by

$$
(v, \alpha)=\frac{1}{2}\left(x_{0}^{r}-x_{0}^{l}+\sigma_{\xi_{0}}, \alpha\right)-(\pi, d \alpha),
$$

where $\alpha$ is a right invariant 1 -form on $G$. It remains to show that $v=0$. Since $\pi$ is multiplicative and since $\xi_{0}$ is $A d_{G}$-invariant, we know that $v$ is multiplicative [L]], i.e., for $g, h \in G$,

$$
v(g h)=l_{g} v(h)+r_{h} v(g) .
$$

Since $v(e)=0$, the fact that $v=0$ follows from the fact that the linearization of $v$ at $e$ is zero. The case for $\mu^{l}$ is similarly proved. 
Remark 4.9 1) The vector fields $x_{0}^{l}$ and $x_{0}^{r}$ are Poisson vector fields because $x_{0}$ is a character of $\mathfrak{g}^{*}$. Let $f_{0}$ be the function on $G$ defined by

$$
f_{0}(g):=\operatorname{det}\left(A d_{g} \text { on } \wedge^{\text {top }} \mathfrak{g}\right)
$$

Then $d\left(\log f_{0}\right)=\xi_{0}^{r}$. Thus

$$
\sigma_{\xi_{0}}=\tilde{\pi}\left(d\left(\log f_{0}\right)\right)
$$

is a Hamiltonian vector field.

2) If we identify the cotangent bundle $T^{*} G$ with the trivial bundle $G \times \mathfrak{g}^{*}$ by right translations, the Lie algebroid structure on $T^{*} G$ becomes that of the transformation Lie algebroid defined by the infinitesimal right dressing action of $\mathfrak{g}^{*}$ on $G$ (see [L]]). Proposition 4.8 can then also be proved as a corollary of Example 3.13 .

Assume now that $H \subset G$ is a connected and closed Poisson Lie subgroup of $G$ [STS. Recall that this is equivalent to $\mathfrak{h}^{\perp} \subset \mathfrak{g}^{*}$ being an ideal in $\mathfrak{g}^{*}$, where

$$
\mathfrak{h}^{\perp}=\left\{\xi \in \mathfrak{g}^{*}:(\xi, x)=0 \forall x \in \mathfrak{h}\right\}
$$

The quotient space $G / H$ has a unique Poisson structure such that the projection map

$$
j: G \rightarrow G / H: g \longmapsto g H
$$

is a Poisson map. Assume that there exists a $G$-invariant volume form $\mu_{0}$ on $G / H$. We wish to determine the modular vector field $\theta_{\mu_{0}}$ on $G / H$ defined by $\mu_{0}$.

Set $\tilde{\mu}_{0}=j^{*} \mu_{0}$. It is a left invariant $l$-form on $G$, where $l=\operatorname{dim}(G / H)$. Choose any $\mu_{1} \in \wedge^{n-l} \mathfrak{g}^{*}$, where $n=\operatorname{dim} G$, such that $\mu=\mu_{1} \wedge \tilde{\mu}_{0}$ is a left invariant volume form on $G$. We have shown that the modular vector field $\theta_{\mu}$ on $G$ defined by $\mu$ is given by (49).

Proposition 4.10 The modular vector field $\theta_{\mu_{0}}$ on $G / H$ defined by $\mu_{0}$ is given by

$$
\theta_{\mu_{0}}=j_{*}\left(\theta_{\mu}\right)=\frac{1}{2} j_{*}\left(x_{0}^{l}+x_{0}^{r}+\sigma_{\xi_{0}}\right)
$$

i.e., it is the projection to $G / H$ by $j$ of the modular vector field $\theta_{\mu}$ on $G$ defined by $\mu=$ $\mu_{1} \wedge \tilde{\mu}_{0}$ 
Proof. Let $\gamma_{1}, \ldots, \gamma_{l}$ be a basis for $\mathfrak{h}^{\perp} \subset \mathfrak{g}^{*}$. Denote by the same letters the corresponding left invariant 1-forms on $G$. Assume that $\mu_{1}=\xi_{1} \wedge \xi_{2} \wedge \cdots \wedge \xi_{t}$, where $t=\operatorname{dim} H$ and the $\xi_{i}$ 's are in $\mathfrak{g}^{*}$ and denote also the corresponding left invariant 1-forms on $G$.

Let $\alpha$ be an arbitrary 1 -form on $G / H$. Let $\tilde{\alpha}=j^{*} \alpha$ be the pull-back of $\alpha$ to $G$ by the projection map $j$. Then

$$
\{\tilde{\alpha}, \mu\}=\left\{\tilde{\alpha}, \mu_{1}\right\} \wedge \tilde{\mu}_{0}+\mu_{1} \wedge\left\{\tilde{\alpha}, \tilde{\mu}_{0}\right\}
$$

Now,

$$
\left\{\tilde{\alpha}, \mu_{1}\right\}=\sum_{j=1}^{t} \xi_{1} \wedge \cdots \wedge \xi_{j-1} \wedge\left\{\tilde{\alpha}, \xi_{j}\right\} \wedge \xi_{j+1} \wedge \cdots \wedge \xi_{t} .
$$

Write $\tilde{\alpha}=\sum_{i=1}^{l} f_{i} \gamma_{i}$, where the $f_{i}$ 's are functions on $G$. Then for each $j=1, \ldots, t$,

$$
\left\{\tilde{\alpha}, \xi_{j}\right\}=\sum_{i}\left(f_{i}\left\{\gamma_{i}, \xi_{j}\right\}-\left(\tilde{\pi}\left(\xi_{j}\right) \cdot f_{i}\right) \gamma_{i}\right)
$$

Since $\mathfrak{h}^{\perp}$ is an ideal of $\mathfrak{g}^{*}$, we have $\left\{\gamma_{i}, \xi_{j}\right\} \in \mathfrak{h}^{\perp}$. Thus

$$
\left\{\tilde{\alpha}, \xi_{j}\right\} \wedge \tilde{\mu}_{0}=0, \forall j
$$

and

$$
\left\{\tilde{\alpha}, \mu_{1}\right\} \wedge \tilde{\mu}_{0}=0
$$

Hence

$$
\{\tilde{\alpha}, \mu\}=\mu_{1} \wedge\left\{\tilde{\alpha}, \tilde{\mu}_{0}\right\}=\mu_{1} \wedge j^{*}\left\{\alpha, \mu_{0}\right\} .
$$

Therefore, using $\pi_{G}$ and $\pi$ to denote respectively the Poisson bi-vector fields on $G$ and on $G / H$, we have from the definition that

$$
\begin{aligned}
\theta_{\mu}(\tilde{\alpha}) \mu & =\left\{\tilde{\alpha}, \mu_{1} \wedge \tilde{\mu}_{0}\right\}-\left(\pi_{G}, d \tilde{\alpha}\right) \mu_{1} \wedge \tilde{\mu}_{0} \\
& =\mu_{1} \wedge j^{*}\left\{\alpha, \mu_{0}\right\}-j^{*}(\pi, d \alpha) \mu_{1} \wedge \tilde{\mu}_{0} \\
& =\mu_{1} \wedge j^{*}\left(\theta_{\mu_{0}}(\alpha) \mu_{0}\right) \\
& =j^{*}\left(\theta_{\mu_{0}}(\alpha)\right) \mu
\end{aligned}
$$

Thus

$$
\theta_{\mu}(\tilde{\alpha})=j^{*}\left(\theta_{\mu_{0}}(\alpha)\right) .
$$

This shows that $j_{*} \theta_{\mu}$ is well-defined and that

$$
\theta_{\mu_{0}}=j_{*} \theta_{\mu}=\frac{1}{2} j_{*}\left(x_{0}^{l}+x_{0}^{r}+\sigma_{\xi_{0}}\right) .
$$


Q.E.D.

Example 4.11 Let $K$ be a compact semi-simple Lie group and let $T$ be a maximal torus of $K$. There is a naturally defined Poisson structure on $K$ making it into a Poisson Lie group [L-W]. This Poisson structure vanishes at points in $T$. Thus it descends to a Poisson structure, called the Bruhat-Poisson structure, on the flag manifold $K / T$. It follows from Proposition 4.10 that the modular vector field of the Bruhat-Poisson structure defined by a $K$-invariant volume form on $K / T$ is the vector field defined by $2 i H_{\rho}$ in the Lie algebra $\mathfrak{t}$ of $T$, where $\rho$ is half of the sum of all the positive roots, and $2 i H_{\rho}$ denotes the element in $\mathfrak{t}$ corresponding to $2 i \rho$ under the identification of $\mathfrak{t}$ and $\mathfrak{t}^{*}$ via the Killing form.

\section{A cohomology pairing}

Let $A$ be a Lie algebroid over $P$ with anchor map $\rho: A \rightarrow T P$. In this section, we show that there is a natural pairing between $H^{k}(A)$, the $k$-th Lie algebroid cohomology of $A$ with trivial coefficients, and $H^{r-k}\left(A, Q_{A}\right)$, the $(r-k)$-th Lie algebroid cohomology of $A$ with coefficients in $Q_{A}=\wedge^{r} A \otimes \wedge^{t o p} T^{*} P$, where $r$ is the rank of $A$, and $0 \leq k \leq r$. For simplicity, we assume that $P$ is compact and orientable. For a general $P$, we need to consider the "compactly supported" Lie algebroid cohomology of $A$ and replace $\wedge^{t o p} T^{*} P$ by $\left|\wedge^{\text {top }} T^{*} P\right|$, the density bundle of $P$.

Recall that

$$
H^{k}(A)=H^{k}\left(C, d_{A}\right), \quad H^{k}\left(A, Q_{A}\right)=H^{k}\left(\tilde{C}, \tilde{d}_{A}\right),
$$

where $C=\oplus_{k=0}^{r} C^{k}=\oplus_{k=0}^{r} \Gamma\left(\wedge^{k} A^{*}\right)$ with $d_{A}$ given by (3), and $\tilde{C}=C \otimes \Gamma\left(Q_{A}\right)$ with $\tilde{d}_{A}: \tilde{C}^{k} \rightarrow \tilde{C}^{k+1}$ given by

$$
\tilde{d}_{A}(\xi \otimes s)=d_{A} \xi \otimes s+(-1)^{|\xi|} \xi \otimes D s,
$$

where, for $s=X \otimes \mu \in \Gamma\left(Q_{A}\right), D s \in \Gamma\left(A^{*}\right) \otimes \Gamma\left(Q_{A}\right)$ maps a section $a$ of $A$ to the section

$$
D_{a}(s)=[a, X] \otimes \mu+X \otimes L_{\rho(a)} \mu
$$

of $Q_{A}($ see $(31))$.

Fix an orientation of $P$. Then integrating with respect to this orientation gives rise to the following non-degenerate pairing between $C^{k}$ and $\tilde{C}^{r-k}$ :

$$
((\xi, \eta \otimes X \otimes \mu)) \stackrel{\text { def }}{=} \int(\xi \wedge \eta, X) \mu \text {. }
$$


Theorem 5.1 For $\xi \in C^{k-1}$ and $\eta \otimes X \otimes \mu \in \tilde{C}^{r-k}$,

$$
\left(\left(d_{A} \xi, \eta \otimes X \otimes \mu\right)\right)+(-1)^{|\xi|}\left(\left(\xi, \tilde{d}_{A}(\eta \otimes X \otimes \mu)\right)\right)=0 .
$$

Consequently, there is an induced pairing between $H^{k}(A)$ and $H^{n-k}\left(A, Q_{A}\right)$.

Proof. Consider $c=(\xi \wedge \eta) \otimes X \otimes \mu \in \tilde{C}^{r-1}$. We know that

$$
\tilde{d}_{A}(c)=d_{A} \xi \otimes(\eta \otimes X \otimes \mu)+(-1)^{|\xi|} \xi \wedge \tilde{d}_{A}(\eta \otimes X \otimes \mu) \in \tilde{C}^{r} .
$$

Theorem 5.1 now follows from the following Stokes' Theorem.

Q.E.D.

Theorem 5.2 (Stokes' Theorem) Identify $\tilde{C}^{r}=\Gamma\left(\wedge^{r} A^{*} \otimes \wedge^{r} A \otimes \wedge^{\text {top }} T^{*} P\right)$ with the space of top-degree forms on $P$ by pairing the factors in $\wedge^{r} A^{*}$ and $\wedge^{r} A$ pointwise. We have, for every $c=\xi \otimes X \otimes \mu \in \tilde{C}^{r-1}$,

$$
\left.\left.\tilde{d}_{A}(c)=(-1)^{r-1} d(\rho(\xi\lrcorner X)\right\lrcorner \mu\right) .
$$

Consequently,

$$
\int_{P} \tilde{d}_{A}(c)=0
$$

Proof. We only need to prove (53) locally. Let $U$ be an open subset of $P$ over which both $\wedge^{r} A$ and $\wedge^{t o p} T^{*} P$ are trivial with nowhere vanishing sections $X_{0}$ and $\mu_{0}$ respectively. Set $s_{0}=X_{0} \otimes \mu_{0}$. Let $\theta_{0}$ be the section of $A^{*}$ over $U$ such that

$$
\theta_{0}(a) s_{0}=D_{a} s_{0}
$$

for every section $a$ of $A$. Write $X \otimes \mu=f X_{0} \otimes \mu_{0}=f s_{0}$ over $U$. We have,

$$
\tilde{d}_{A}(c)=\left(d_{A}(f \xi)+\theta_{0} \wedge f \xi, X_{0}\right) \mu_{0} .
$$

Set $a=f \xi\lrcorner X_{0}$. It follows from the definitions of $\theta_{0}$ and the representation of $A$ on $Q_{A}$ that

$$
\left(\theta_{0} \wedge f \xi, X_{0}\right) \mu_{0}=-\left(d_{A}(f \xi), X_{0}\right) \mu_{0}+(-1)^{r-1} L_{\rho(a)} \mu_{0}
$$

Hence,

$$
\tilde{d}_{A}(c)=(-1)^{r-1} L_{\rho(a)} \mu=(-1)^{r-1} d(\rho(\xi \sqcup X) \downarrow \mu) .
$$

This proves (53). It now follows from Stokes' Theorem for de Rham cohomology that

$$
\int_{P} \tilde{d}_{A}(c)=0
$$




\section{Q.E.D.}

Corollary 5.3 Let $P$ be an orientable Poisson manifold with a fixed orientation. For a differential form $\alpha$ and a compactly supported differential form $\beta$, define

$$
(\alpha, \beta)=\int_{P} \alpha \wedge \beta
$$

Let $\partial_{\pi}: \Omega^{k}(P) \rightarrow \Omega^{k-1}(P)$ be the Koszul-Brylinski operator as defined by (38). Then, for any forms $\alpha$ and $\beta$ with $|\alpha|+|\beta|-1=n=\operatorname{dim} P$, we have

$$
\left(\partial_{\pi} \alpha, \beta\right)+(-1)^{(|\alpha|-1)}\left(\alpha, \partial_{\pi} \beta\right)=0 .
$$

Thus we get an induced pairing between the Poisson homology spaces $H_{k}(P)$ and $H_{n-k}(P)$.

Proof. Fix a volume form $\mu_{0}$ of $P$. Let $\theta_{0}$ be the modular vector field defined by $\mu_{0}$. Let $U \in \chi^{n-|\alpha|}(P)$ and $V \in \chi^{n-|\beta|}(P)$ be such that

$$
\alpha=U\lrcorner \mu_{0}, \quad \beta=V \downarrow \mu_{0} .
$$

let (( )) be the pairing on $\chi(P)$ given by

$$
\left(\left(V_{1}, V_{2}\right)\right)=\int_{P}\left(V_{1} \wedge V_{2}, \mu_{0}\right) \mu_{0}
$$

Then we know from Theorem 5.1 that

$$
(([\pi, U], V))+(-1)^{|U|}\left(\left(U,[\pi, V]+2 \theta_{0} \wedge V\right)\right)=0 .
$$

It follows that

$$
\begin{aligned}
& \left(\left([\pi, U]+\theta_{0} \wedge U, V\right)\right)+(-1)^{|U|}\left(\left(U,[\pi, V]+\theta_{0} \wedge V\right)\right) \\
= & (([\pi, U], V))+(-1)^{|U|}((U,[\pi, V]))+\left(\left(\theta_{0} \wedge U, V\right)\right)+(-1)^{|U|}\left(\left(U, \theta_{0} \wedge V\right)\right) \\
= & (([\pi, U], V))+(-1)^{|U|}((U,[\pi, V]))+2(-1)^{|U|}\left(\left(U, \theta_{0} \wedge V\right)\right) \\
= & (([\pi, U], V))+(-1)^{|U|}\left(\left(U,[\pi, V]+2 \theta_{0} \wedge V\right)\right) \\
= & 0 .
\end{aligned}
$$

From Theorem 4.5, we have

$$
\begin{aligned}
& \left(\partial_{\pi} \alpha, \beta\right)=(-1)^{|U|+1}\left(\left([\pi, U]+\theta_{0} \wedge U, V\right)\right) \\
& \left(\alpha, \partial_{\pi} \beta\right)=(-1)^{|V|+1}\left(\left(U,[\pi, V]+\theta_{0} \wedge V\right)\right) .
\end{aligned}
$$

Thus we get (55). 
We now turn to the discussion of the non-degeneracy of the pairing $(()$,$) between H^{k}(A)$ and $H^{r-k}\left(A, Q_{A}\right)$. We will see that it is not always non-degenerate. We first look at some familiar examples.

Example 5.4 When $A$ is a Lie algebra $\mathfrak{g}$ considered as a Lie algebroid over a one point space, we have $Q_{A}=\wedge^{r} \mathfrak{g}$, where $r=\operatorname{dim} \mathfrak{g}$. The pairing (( , )) in Theorem 5.1 is nondegenerate and it gives rise to an isomorphism

$$
H^{k}(\mathfrak{g})^{*} \cong H^{r-k}\left(\mathfrak{g}, \wedge^{r} \mathfrak{g}\right)
$$

This is the familiar Poincare duality for Lie algebra cohomology.

Example 5.5 Let $P$ be a compact orientable manifold and let $A=T P$ be the tangent bundle Lie algebroid with the identity anchor map. The line bundle $Q_{A}$ is trivial and so is the representation of $A$ on $Q_{A}$. The pairing $(()$,$) in Theorem 5.1$ is the one obtained by integrating the wedge product of $k$-forms and $(n-k)$-forms, where $n=\operatorname{dim} P$. This, of course, is non-degenerate and gives the well-known Poincare duality for de Rham cohomology of $P$.

Example 5.6 Let $A$ be an arbitrary vector bundle over $P$ of rank $r$. Consider $A$ as a Lie algebroid over $P$ with the zero Lie bracket on its sections and the zero anchor map to $T P$. Then $H^{k}(A)$ is the space of smooth sections of $\wedge^{k} A^{*}$, and $H^{r-k}\left(A, Q_{A}\right)$ is the space of smooth sections of

$$
\wedge^{r-k} A^{*} \otimes \wedge^{r} A \otimes \wedge^{t o p} T^{*} P \cong \wedge^{k} A \otimes \wedge^{t o p} T^{*} P
$$

The pairing between these two spaces, pairing the elements in $\wedge^{k} A^{*}$ and in $\wedge^{k} A$ pointwise and integrating the resulting top degree form on $P$ over $P$, is again non-degenerate in each argument.

Example 5.7 We now consider the case when $A=P \times \mathfrak{g}$ is a transformation Lie algebroid defined by a Lie algebra homomorphism $\rho: \mathfrak{g} \rightarrow \chi^{1}(P)$ from a Lie algebra $\mathfrak{g}$ to the Lie algebra of vector fields on $P$. See Example 3.4. In this case, both $H^{\bullet}(A)$ and $H^{\bullet}\left(A, Q_{A}\right)$ 
are natually isomorphic to certain Lie algebra cohomology spaces of $\mathfrak{g}$, and the pairing $(()$, in Theorem 5.1 is the one that occurs in the Poincare duality of Lie algebra cohomology (see, for example, $[\mathrm{Kp}]$ ). More precisely, we have

$$
H^{k}(A) \cong H^{k}\left(\mathfrak{g}, C^{\infty}(P)\right)
$$

where the right hand side is the Lie algebra cohomology of $\mathfrak{g}$ with coefficients in $C^{\infty}(P)$ considered as a $\mathfrak{g}$-module via $\rho$ :

$$
x \cdot f=\rho(x) \cdot f, \quad x \in \mathfrak{g}, f \in C^{\infty}(P),
$$

and

$$
H^{r-k}\left(A, Q_{A}\right) \cong H^{r-k}\left(\mathfrak{g}, \wedge^{r} \mathfrak{g} \otimes \Omega^{t o p}(P)\right),
$$

where $r=\operatorname{dim} \mathfrak{g}$ is the rank of $A$, and the right hand side is the Lie algebra cohomology of $\mathfrak{g}$ with coefficients in the tensor product module $\wedge^{r} \mathfrak{g} \otimes \Omega^{\text {top }}(P)$. The space $\wedge^{r} \mathfrak{g}$ is equipped with the adjoint representation of $\mathfrak{g}$, and $\Omega^{t o p}(P)$ is equipped with the action of $\mathfrak{g}$ by Lie derivatives:

$$
x \cdot \mu=L_{\rho(x)} \mu .
$$

The isomorphisms (56) and (58) are easily seen to come from isomorphisms on the complex level:

$$
\begin{aligned}
& C^{k}=\Gamma\left(\wedge^{k} A^{*}\right) \cong \wedge^{k} \mathfrak{g}^{*} \otimes C^{\infty}(P) \\
& \tilde{C}^{r-k}=\Gamma\left(\wedge^{r-k} A^{*} \otimes \wedge^{r} A \otimes \wedge^{t o p} T^{*} P\right) \cong \wedge^{r-k} \mathfrak{g}^{*} \otimes \wedge^{r} \mathfrak{g} \otimes \Omega^{t o p}(P) .
\end{aligned}
$$

Consider the pairing between $C^{\infty}(P)$ and $\Omega^{t o p}(P)$ given by

$$
(f, \mu)=\int_{P} f \mu \text {. }
$$

For each $x \in \mathfrak{g}$, it follows from

$$
\int_{P} L_{\rho(x)}(f \mu)=0
$$

that

$$
(x \cdot f, \mu)+(f, x \cdot \mu)=0 .
$$

Thus, the two $\mathfrak{g}$-modules $C^{\infty}(P)$ and $\Omega^{t o p}(P)$ are contragradient to each other with respect to the above pairing. Under the isomorphisms above, the pairing (( )) in Theorem 5.1 becomes the one between $\wedge^{k} \mathfrak{g}^{*} \otimes C^{\infty}(P)$ and $\wedge^{r-k} \mathfrak{g}^{*} \otimes \wedge^{r} \mathfrak{g} \otimes \Omega^{\text {top }}(P)$ given by

$$
\left(\xi \otimes f, \eta \otimes X_{0} \otimes \mu\right)=\left(\xi \wedge \eta, X_{0}\right)(f, \mu) .
$$


This is exactly the pairing that occurs in the Poincare duality for Lie algebra cohomology.

In this example, even though the pairing between the two $\mathfrak{g}$-modules $C^{\infty}(P)$ and $\Omega^{\text {top }}(P)$ is non-degenerate, they are not the full duals of each other, so we can not conclude that the induced pairing on the cohomology spaces is non-degenerate. For example,

$$
H^{0}\left(\mathfrak{g}, C^{\infty}(P)\right)=C^{\infty}(P)^{\mathfrak{g}}
$$

and

$$
H^{r}\left(\mathfrak{g}, \wedge^{r} \mathfrak{g} \otimes \Omega^{t o p}(P)\right) \cong \frac{\Omega^{t o p}(P)}{\mathfrak{g} \cdot \Omega^{t o p}(P)} .
$$

These two spaces are not necessarily dual to each other, as we see from the following example: the vector field

$$
v_{0}=\left(1-e^{i \theta}\right)^{N} \frac{d}{d \theta},
$$

where $N \geq 2$ is an integer, defines an action of the 1-dimensional Lie algebra $\mathfrak{g}=\mathbb{R}^{1}$ on $P=S^{1}$. The space $H^{0}\left(\mathfrak{g}, C^{\infty}(P)\right)$ is 1-dimensional, while the space $H^{r}\left(\mathfrak{g}, \wedge^{r} \mathfrak{g} \otimes \Omega^{t o p}(P)\right)$ is at least $N$-dimensional.

Example 5.8 For a general Lie algebroid $A$ over $P$, we have

$$
H^{0}(A) \cong\left\{f \in C^{\infty}(P): \rho(a) \cdot f=0, \forall a \in \Gamma(A)\right\}
$$

and, from Stokes' Theorem,

$$
H^{r}\left(A, Q_{A}\right) \cong \frac{\Omega^{t o p}(P)}{\left\{L_{\rho(a)} \mu, a \otimes \mu \in \Gamma(A) \otimes \Omega^{t o p}(P)\right\}}
$$

If $P$ is not compact, we consider compactly supported top-degree forms in $H^{r}\left(A, Q_{A}\right)$. The following is another example where the two spaces $H^{0}$ and $H^{r}$ are not dual to each other: let $P=\mathbb{R}^{2}$ with the Poisson structure given by

$$
\{x, y\}=\left(x^{2}+y^{2}\right)^{N}
$$

where $N$ is an integer and $N \geq 3$. Let $A$ be $T^{*} P$ with the cotangent bundle Lie algebroid defined by this Poisson structure. Again, $H^{0}(A)$ is 1 -dimensional and $H^{r}\left(A, Q_{A}\right)$ is at least $(N-1)$-dimensional. 


\section{The holomorphic case}

In this section, we extend our results to the holomorphic setting. Closely related results are studied in [B-Z] in different language, where questions of monodromy are also discussed.

The theory of Lie algebroids can be developed in the setting of sheaves (see [B-B] [K-T]). Let $O_{P}$ be either the sheaf of smooth functions on a manifold or the sheaf of holomorphic functions on a complex manifold (one can also consider the algebraic setting, but it will not be useful for us). Let $T P$ be the tangent sheaf defined over $O_{P}$. Note that $T P$ is a sheaf of Lie algebras over the scalars, where the Lie algebra structure on sections over an open set is the usual Lie bracket of vector fields.

A Lie algebroid $A$ over $O_{P}$ is a sheaf of $O_{P}$ modules together with 1) a Lie algebra structure on $A$ making $A$ into a sheaf of Lie algebras over the scalars, and 2) a homomorphism $\rho: A \rightarrow T P$ of sheaves of modules over $O_{P}$ and of Lie algebras, such that for $f \in O_{P}(U)$ and $\omega_{1}, \omega_{2} \in A(U)$, the following derivation law holds:

$$
\left\{\omega_{1}, f \omega_{2}\right\}=f\left\{\omega_{1}, \omega_{2}\right\}+\left(\rho\left(\omega_{1}\right) f\right) \omega_{2} .
$$

As before, $\rho$ is called the anchor map of the Lie algebroid. For the rest of this section, we will always assume that $A$ is locally free, so that it is the sheaf of sections of a vector bundle over $O_{P}$.

A representation of $A$ is a sheaf $M$ of $A$ modules, i.e., for every open set $U$, the space of sections $M(U)$ is a representation of $A(U)$ satisfying the properties given in Section 1 and the representations are compatible with restriction maps. It follows as in Section 6 that the line bundle $Q_{A}=\wedge^{\text {top }} A \otimes \wedge^{\text {top }} T^{*} P$ determines an $A$ module.

Consider the sheaf $\Omega^{k}(A)$ of sections of $\wedge^{k} A^{*}$. The formula for $d_{A}$ given in Section 2 makes the sequence

$$
\ldots \rightarrow \Omega^{k-1}(A) \rightarrow \Omega^{k}(A) \rightarrow \Omega^{k+1}(A) \rightarrow \ldots
$$

into a complex of sheaves. We can take the hypercohomology of this complex of sheaves $\mathbb{H}^{\bullet}\left(\Omega^{\bullet}(A)\right)$ (see G-H] p. 445). We define Lie algebroid cohomology

$$
H^{\bullet}\left(A, O_{P}\right)=\mathbb{H}^{\bullet}\left(\Omega^{\bullet}(A)\right)
$$

We also consider the cohomology sheaves $\mathcal{H}^{\bullet}\left(A, O_{P}\right)$. To define these sheaves, first consider the cohomology of the above complex on any open set. The assignment

$$
U \mapsto H^{i}\left(\Omega^{\bullet}(A)(U), d_{A}\right)
$$


determines a presheaf. By definition, $\mathcal{H}^{\bullet}\left(A, O_{P}\right)$ is the sheaf associated to this presheaf.

We will compute $H^{\bullet}(P, \mathcal{S})$ for a sheaf $\mathcal{S}$ using Cech cohomology, so $H^{i}\left(A, O_{P}\right)$ is the total cohomology of the double complex $C^{p}\left(P, \Omega^{q}(A)\right)$. The usual filtrations give two first quadrant spectral sequences converging to $H^{\bullet}\left(A, O_{P}\right)$ G-H. The first has $E_{2}$ term $H^{p}\left(P, \mathcal{H}^{q}\left(A, O_{P}\right)\right)$ and the second has $E_{2}$ term $H^{q}\left(H^{p}\left(P, \Omega^{\bullet}(A)\right)\right)$.

In the case where $O_{P}$ is smooth functions on $P$, we have $\left.H^{p}\left(P, \Omega^{\bullet}(A)\right)\right)=0$ for $p>0$ since the sheaves $\Omega^{\bullet}(A)$ are soft. It follows that in this case the second spectral sequence degenerates at the $E_{2}$ term, so that $H^{q}\left(A, O_{P}\right)=H^{q}\left(\Gamma\left(P, \Omega^{\bullet}(A)\right)\right)$. Thus our hypercohomology definition of Lie algebroid cohomology agrees with the definition given in Section 2 . The same remarks apply when $O_{P}$ is the sheaf of holomorphic functions on a Stein manifold $P$.

We return to the general case. We can compute Cech cohomology using an open cover $U_{\alpha}$ of $P$ such that all intersections of the $U_{\alpha}$ 's are contractible or empty. In particular, we may assume that on an intersection of open sets, every nowhere vanishing function has a logarithm. On such a cover, $Q_{A}$ is a trivial line bundle. We can define a modular class $\Theta_{A} \in H^{0}\left(P, \mathcal{H}^{1}\left(A, O_{P}\right)\right)$ as follows. On each open set $U_{\alpha}$ in our cover, choose a nowhere vanishing section $s_{\alpha}$ of $Q_{A} \mid U_{\alpha}$. Define a modular class on $U_{\alpha}$ by setting

$$
D_{a} s_{\alpha}=\Theta_{s_{\alpha}}(a) s_{\alpha}
$$

As in Section 3, it follows that the class of $\Theta_{s_{\alpha}} \in \mathcal{H}^{1}\left(A, O_{P}\right)$ is independent of the choice of $s_{\alpha}$. We will denote it by $\Theta_{\alpha}$. Moreover, $\Theta_{\alpha}$ and $\Theta_{\beta}$ agree on open sets $U_{\alpha}$ and $U_{\beta}$ in our cover. Indeed, on $U_{\alpha} \cap U_{\beta}$, we know $s_{\alpha}=g_{\alpha \beta} s_{\beta}$ for some nowhere vanishing function $g_{\alpha \beta}$. Then it follows as above that the cohomology classes $\Theta_{\alpha}$ and $\Theta_{\beta}$ coincide. Thus we have a well-defined global section of the sheaf $\mathcal{H}^{1}\left(A, O_{P}\right)$ which we denote by $\Theta_{A}$. In fact, the functions $g_{\alpha \beta}$ are just the transition functions of $Q_{A}$.

We wish to determine whether $\Theta_{A}$ determines a class in $H^{1}\left(A, O_{P}\right)$. We showed in the previous paragraph that $\Theta_{A}$ determines a class in $H^{0}\left(P, \mathcal{H}^{1}\left(A, O_{P}\right)\right)$, which is in $E_{2}^{0,1}$ for the first spectral sequence discussed above. $\Theta_{A}$ defines a class in $H^{1}\left(A, O_{P}\right)$ if it is annihilated by all differentials in the spectral sequence. All differentials $d_{r}$ for $r>2$ annihilate $\Theta_{A}$ for reasons of degree. Thus it suffices to determine if $d_{2} \Theta_{A}=0$ for the differential

$$
d_{2}: H^{0}\left(P, \mathcal{H}^{1}\left(A, O_{P}\right)\right) \rightarrow H^{2}\left(P, \mathcal{H}^{0}\left(A, O_{P}\right)\right)
$$

Denote the Cech differential by $\delta$. By definition, for a class $r \in H^{0}\left(P, \mathcal{H}^{1}\left(A, O_{P}\right)\right), d_{2}(r)=$ 
$\delta c$, where $c \in C^{1}\left(P, \mathcal{H}^{0}\left(A, O_{P}\right)\right)$ is chosen so $d_{A} c=\delta r$ (see [B-T]).

Proposition 6.1 Let $i: \mathbb{C}_{P} \rightarrow \mathcal{H}^{0}\left(A, O_{P}\right)$ be the inclusion of the constant sheaf into the sheaf of functions annihilated by $A$. Then

$$
d_{2}\left(\Theta_{A}\right)=i_{*}\left(2 \pi \sqrt{-1} c_{1}\left(Q_{A}\right)\right)
$$

where $c_{1}\left(Q_{A}\right)$ is the first Chern class of the line bundle $Q_{A}$ in the holomorphic setting and of the complexification of $Q_{A}$ in the real setting which is 0 .

Proof. We defined $\Theta_{A}$ by choosing a nowhere vanishing section $s_{\alpha} \in \Gamma\left(U_{\alpha}, Q_{A}\right)$. Then

$$
\delta \Theta_{A}(a)\left(U_{\alpha} \cap U_{\beta}\right)=\left.\frac{D_{a}\left(s_{\alpha}\right)}{s_{\alpha}}\right|_{U_{\alpha} \cap U_{\beta}}-\left.\frac{D_{a}\left(s_{\beta}\right)}{s_{\beta}}\right|_{U_{\alpha} \cap U_{\beta}}=d_{A}\left(\log \left(g_{\alpha \beta}\right)\right)(a)
$$

where the $g_{\alpha \beta}$ are the transition functions of $Q_{A}$ discussed above. Then

$$
d_{2}\left(\Theta_{A}\right)\left(U_{\alpha} \cap U_{\beta} \cap U_{\gamma}\right)=\log \left(g_{\alpha \beta}\right)+\log \left(g_{\beta \gamma}\right)-\log \left(g_{\alpha \gamma}\right)
$$

This is, up to a factor of $2 \pi \sqrt{-1}$, the usual sheaf cohomology description of the first Chern class of the line bundle $Q_{A}$. Since we regard this class as an element of $H^{2}\left(P, \mathcal{H}^{0}\left(A, O_{P}\right)\right)$, we write it as $i_{*}\left(2 \pi \sqrt{-1} c_{1}\left(Q_{A}\right)\right)$.

\section{Q.E.D.}

Corollary 6.2 Suppose $c_{1}\left(Q_{A}\right)=0$. Then $d_{2}\left(\Theta_{A}\right)=0$.

When $d_{2}\left(\Theta_{A}\right)=0$, we denote the corresponding class in $H^{1}\left(A, O_{P}\right)$ by $\theta_{A}$.

We remark that it follows from the proposition that $d_{2}\left(\Theta_{A}\right)=0$ when $O_{P}$ is the sheaf of smooth functions. Indeed, in this case, the line bundle $Q_{A}$ has an underlying real structure so $c_{1}\left(Q_{A}\right)=0(\overline{\mathrm{B}-\mathrm{T}})$. In the holomorphic setting, $Q_{A}$ is always trivial if $P$ is a Stein manifold. In addition, if $P$ admits a holomorphic symplectic structure, then $\wedge^{\text {top }} T^{*} P$ is trivial, so $c_{1}\left(Q_{T^{*} P}\right)=0$. This is the case for $G / H$, where $G$ is a complex reductive group and $H$ is a maximal torus, and a Poisson structure analogous to that of [L-W] can be introduced. In addition, in the case where the anchor map is zero, $d_{2}\left(\Theta_{A}\right)=0$ even though $c_{1}\left(Q_{A}\right)$ may not vanish. 


\section{Appendix A: The adjoint "representation" of a Lie alge- broid}

In this appendix, we will describe a construction which includes as special cases the adjoint representation of a Lie algebra and the (dual of the) flat "Bott" connection on the normal bundle to a foliation. Given a Lie algebroid $A$, we will construct a "representation up to homotopy" of $A$ on the "formal difference" $A \ominus T P$. Taking the highest exterior power of this object will yield the representation of $A$ on $Q_{A}$ described in Section 3. That is all we will use of our construction in this paper, but we believe that it is interesting in its own right.

The idea behind our construction is similar to that in the construction of the representation of $A$ on $Q_{A}$, namely that neither the Lie derivative action of $A$ on itself nor on $T P$ is a representation, but that "the anomalies cancel." The following discussion makes this idea precise.

Definition 7.1 Let $A$ be a Lie algebroid over $P$ and let $(E, \partial)$ be a bundle of $\mathbb{Z}_{2}$-graded complexes over $P$; i.e., $E$ is a $\mathbb{Z}_{2}$-graded vector bundle over $P$ and $\partial$ is a bundle map of degree 1 with $\partial^{2}=0$. A representation up to homotopy of $A$ on $(E, \partial)$ is an $\mathbb{R}$-bilinear map

$$
\Gamma(A) \times \Gamma(E) \longrightarrow \Gamma(E): a \otimes s \longmapsto D_{a} s
$$

such that the operators $D_{a}$ preserve the grading and commute with the action of $\partial$ on sections, and such that the properties

$$
\begin{aligned}
& \text { (2) } D_{a}(f s)=f D_{a} s+(\rho(a) f) s \\
& \text { (3) } D_{a}\left(D_{b} s\right)-D_{b}\left(D_{a} s\right)=D_{[a, b]} s
\end{aligned}
$$

of a representation hold, while property

$$
\text { (1) } D_{f a} s=f D_{a} s
$$

holds only up to homotopy, in the sense that for each $a \in \Gamma(A)$ and $f \in C^{\infty}(P)$ there is a bundle map $I(a, f): E \longrightarrow E$ of degree 1 such that

$$
\left(1^{\prime}\right) D_{f a} s=f D_{a} s+I(a, f) \partial s+\partial I(a, f) s .
$$


Remark 7.2 If we drop condition (3) from the definition of a representation, we get the definition of an $A$-connection on a vector bundle $E$. The difference of the left and right hand sides of $(3)$ is then $C^{\infty}(P)$-linear in $a, b$, and $s$ and becomes the curvature tensor of the connection. Similarly, we can drop condition (3) from Definition 7.1 to define an $A$-connection up to homotopy, and we could also weaken (3) to require flatness only up to homotopy in the definition of a representation. Finally, one might put further conditions on the trilinear expressions $I(a, f) s$ as one does in the the theory of strongly homotopy Lie algebras $[\mathrm{L}-\mathrm{M}]$. We have had no need to explore these options yet, though.

For a bundle $(E, \partial)$ of complexes over $P$, the homology $H(E)$ is not a vector bundle unless $\partial$ has constant rank. We can still consider $H(E)$ as a $C^{\infty}(P)$-module by looking at the action of $\partial$ on sections of $E$, though, so that the notion of a representation of $A$ on $H(E)$ still makes sense. Also, we will define the determinant line bundle $\wedge^{\text {top } E}$ of the graded vector bundle $E=E_{0} \oplus E_{1}$ to be $\wedge^{\text {top }} E_{0}^{*} \otimes \wedge^{\text {top }} E_{1}$.

Proposition 7.3 A representation up to homotopy of a Lie algebroid $A$ on a bundle $(E, \partial)$ of $\mathbb{Z}_{2}$-graded complexes induces representations (not just up to homotopy) on the homology

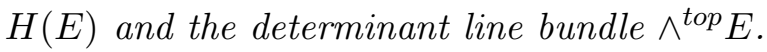

Proof. Since each $D_{a}$ commutes with $\partial$, it induces an operator $H\left(D_{a}\right)$ on homology. Properties (2) and (3) of the $D_{a}$ 's are inherited by the induced operators. To verify (1), we let $s$ be a cycle and find from $\left(1^{\prime}\right)$ that $D_{f a} s-f D_{a} s$ is a boundary.

When $\partial$ has constant rank, the determinant bundle of $H(E)$ is isomorphic to that of $E$, so the second part of the theorem follows from the first part in that special situation. For the general case, we will define and study the extended operators locally.

First of all, we note that a family of operators $D_{a}$ on sections of a vector bundle $V$ satisfying (2) and (3) can be extended in a unique way to a family of operators on all the tensors over $V$, satisfying the same identities, by requiring that the operators be derivations with respect to tensor product and commute with contractions. This is done just is as usually done for Lie derivatives or covariant derivatives. In particular, we obtain operators, also denoted by $D_{a}$, on $E^{*}, \wedge^{t o p} E_{0}^{*}, \wedge^{t o p} E_{1}$, and $\wedge^{t o p} E_{0}^{*} \otimes \wedge^{t o p} E_{1}$.

For instance, the operators on $E^{*}$ are defined by

$$
\left(D_{a} \omega, s\right)=\rho(a) \cdot(\omega, s)-\left(\omega, D_{a} s\right),
$$


from which it follows that we have a representation up to homotopy on the dual complex $\left(E^{*}, \partial^{*}\right)$, using the bundle maps $-I(a, f)^{*}$ to satisfy $\left(1^{\prime}\right)$.

To compute the operators on $\wedge^{t o p} E_{1}$, we use a local basis $t_{1}, \ldots, t_{l}$ for the sections of $E_{1}$. By the derivation property,

$$
D_{a}\left(t_{1} \wedge \ldots \wedge t_{l}\right)=\sum_{r=1}^{l}\left(t_{1} \wedge \ldots \wedge D_{a} t_{r} \wedge \ldots \wedge t_{l}\right) .
$$

Then

$$
\begin{aligned}
D_{f a}\left(t_{1} \wedge \ldots \wedge t_{l}\right) & =\sum_{r=1}^{l}\left(t_{1} \wedge \ldots \wedge D_{f a} t_{r} \wedge \ldots \wedge t_{l}\right) \\
& =f D_{a}\left(t_{1} \wedge \ldots \wedge t_{l}\right) \\
& +\sum_{r=1}^{l}\left(t_{1} \wedge \ldots \wedge\left(I^{10}(a, f) \partial^{01}+\partial^{10} I^{01}(a, f)\right) t_{r} \wedge \ldots \wedge t_{l}\right),
\end{aligned}
$$

where the superscript ${ }^{i j}$ on $I$ or $\partial$ refers to the part of that bundle map which goes from $E_{j}$ to $E_{i}$

Expressing $\left(I^{10}(a, f) \partial^{01}+\partial^{10} I^{01}(a, f)\right) t_{r}$ in terms of our basis, we see that all but its $r$ 'th component is annihilated by another factor in the wedge product, so that we get a simple expression in terms of a trace:

$$
D_{f a}\left(t_{1} \wedge \ldots \wedge t_{l}\right)=f D_{a}\left(t_{1} \wedge \ldots \wedge t_{l}\right)+\operatorname{Tr}\left(I^{10}(a, f) \partial^{01}+\partial^{10} I^{01}(a, f)\right) t_{1} \wedge \ldots \wedge t_{l} .
$$

Similarly, in terms of a basis $s_{1}^{*}, \ldots, s_{k}^{*}$ of local sections of $E_{0}^{*}$, we have:

$$
D_{f a}\left(s_{1}^{*} \wedge \ldots \wedge s_{k}^{*}\right)=f D_{a}\left(s_{1}^{*} \wedge \ldots s_{k}^{*}\right)-\operatorname{Tr}\left(I^{10 *}(a, f) \partial^{01 *}+\partial^{10 *} I^{01 *}(a, f)\right) s_{1}^{*} \wedge \ldots s_{k}^{*} .
$$

Combining the two previous equations and using the derivation property with respect to tensor product, we obtain an expression for the behavior of the $D_{a}$ 's operating on the determinant bundle:

$D_{f a}\left(s_{1}^{*} \wedge \ldots s_{k}^{*} \otimes t_{1} \wedge \ldots \wedge t_{l}\right)=f D_{a}\left(s_{1}^{*} \wedge \ldots s_{k}^{*} \otimes t_{1} \wedge \ldots \wedge t_{l}\right)+K(a, f) s_{1}^{*} \wedge \ldots s_{k}^{*} \otimes t_{1} \wedge \ldots \wedge t_{l}$

where

$$
K(a, f)=\operatorname{Tr}\left(I^{10}(a, f) \partial^{01}+\partial^{10} I^{01}(a, f)\right)-\operatorname{Tr}\left(I^{10 *}(a, f) \partial^{01 *}+\partial^{10 *} I^{01 *}(a, f)\right) .
$$


Using the invariance of the trace under dualization and exchange of factors, we can cancel all the terms in this expression to conclude that $K(a, f)=0$, so that the operation of $A$ on the determinant bundle satisfies $D_{f a}=f D_{a}$, so that we have an honest representation.

\section{Q.E.D.}

With the general notion of representation up to homotopy at hand, we can consider our principal example. Given the Lie algebroid $A$ with anchor $\rho: A \rightarrow T P$, we let $E_{0}=T P$, $E_{1}=A, \partial^{01}=\rho$, and $\partial^{10}=0$. The homology of this little complex is the normal "bundle" $T P / \rho(A)$ to the orbits in degree 0 , and the isotropy "bundle" ker $\rho$ in degree 1 . We call this the normal complex of the Lie algebroid and denote it by $N(A)$. The determinant bundle of the normal complex is precisely what we have called $Q_{A}$.

We define the operators $D_{a}$ on $\Gamma(E)$ for $a \in \Gamma(A)$ by $D_{a} b=[a, b]$ for $b \in \Gamma(A)$ and by $D_{a} u=[\rho(a), u]$ for $u \in \Gamma(T P)$, where the last bracket is the usual bracket of vector fields.

Proposition 7.4 The operators defined above form a representation up to homotopy of the Lie algebroid $A$ on $N(A)$.

Proof. The fact that the $D_{a}$ are chain maps follows from the fact that $\rho$ defines a Lie algebroid homomorphism. Properties (2) and (3) in the definition of a representation are standard facts in the differential calculus on Lie algebroids (see Section 2). For property $\left(1^{\prime}\right)$, we note as in Section 3 that for the action on $A$ we have

$$
D_{f a} b=f D_{a} b-(\rho(b) \cdot f) a
$$

and for the action on $T P$ we have

$$
D_{f a} u=f D_{a} u-(u \cdot f) \rho(a) .
$$

If we define the homotopy operators by $I^{10}(a, f)(u)=-(d f(u)) a$ and $I^{01}(a, f)=0$, we see immediately from the two displayed equations above that $\left(1^{\prime}\right)$ is satisfied, so we have a representation up to homotopy.

\section{Q.E.D.}

When $A$ is a Lie algebra, the operators $D_{a}$ just give the adjoint representation, so we refer to them as the adjoint "representation" in the general case. When $\rho$ is the inclusion of 
a subbundle of $T P$, the homology of the normal complex is just the normal bundle to the corresponding foliation, and we recover the usual flat connection along the leaves. Finally, the associated representation of $A$ on $Q_{A}$ may now be seen as the "top exterior power of the adjoint representation" for a general Lie algebroid.

\section{Appendix B: The adjoint "representation" and modular class of a Lie groupoid}

Let $G$ be a Lie groupoid (i.e. a differentiable groupoid) over $P$. We will denote its target and source maps by $\alpha$ and $\beta$, so that the product $g h$ is defined whenever $\beta(g)=\alpha(h)$. The groupoid analog of the Lie algebroid "representation" constructed in the previous section ought to be a representation up to homotopy of $G$ on the normal complex of its Lie algebroid $A$. There are many possible definitions of the notion of representation up to homotopy for a groupoid, and we have not yet found an optimal one, so we will limit ourselves here to a discussion of the particular case of the adjoint "representation."

Recall that a representation of $G$ on a vector bundle $\lambda: B \rightarrow P$ consists of a mapping $(g, r) \mapsto g r$ from $G \times{ }_{P} B=\{(g, r) \in G \times B \mid \beta(g)=\lambda(r)\}$ to $B$ which is linear on fibres of $B$ and which satisfies the axioms:

$$
\begin{aligned}
& \text { (1) } \lambda(g r)=\alpha(g) \\
& \text { (2) }(g h) r=g(h r) \\
& \text { (3) } e r=r \text { when } e \text { is an identity element of } G \text {. }
\end{aligned}
$$

In the case of Lie algebroids, the distinction between a representation up to homotopy in our sense and an honest representation is that $D_{a} s(x)$ can depend on the entire section $a$ in the former case, while it depends only on $a(x)$ in the latter. In fact, for the adjoint "representation", $D_{a} s(x)$ depends only on the 1-jet of $a$ at $x$, and this carries over to the groupoid case.

We will denote by $J^{1} G$ the 1 -jet prolongation groupoid of the Lie groupoid $G$ over $P$. The elements of $J^{1} G$ are the 1 -jets of bi-sections of $G$, i.e., submanifolds of $G$ which project diffeomorphically to $P$ under the source and target maps.

Unlike the groupoid $G$ itself, $J^{1} G$ has a natural representation on the normal complex $N(A)$ of the Lie algebroid of $G$; i.e., $J^{1} G$ has representations on $A$ and $T P$ for which the anchor $\rho$ is an equivariant map. One way to see this is to consider elements of $J^{1} G$ as special 
subsets of the tangent bundle groupoid $T G$ over $T P$. $T G$ acts on itself by left translations, leaving invariant the part $T_{P} G$ of $T G$ lying over the identity section of $G$. Identifying $T P$ with a subbundle of $T_{P} G$ and $A$ with the normal bundle $T_{P} G / T P$ allows to obtain the required representation of $J^{1} G$.

For example, if $G$ is the pair groupoid $P \times P$, an element $(x, y)$ of $G$ does not naturally transport tangent vectors from $y$ to $x$, but an element of $J^{1} G$ is precisely a vector space isomorphism from $T_{y} P$ to $T_{x} P$.

We would like to make the action of $J^{1} G$ descend to $G$ via the natural "forgetful" projection $j: J^{1} G \rightarrow G$. As the example above shows, this is not possible, but it turns out to be possible "up to homotopy" in the sense that, if $g_{1}^{\prime}$ and $g_{2}^{\prime}$ are two elements of $J^{1} G$ lying over the same $g \in G$, then the mappings induced by the $g_{i}^{\prime}$ between the complexes $N_{\alpha(g)} A$ and $N_{\beta(g)} A$ are homotopic. Proving the latter statement may be reduced to the case where $g$ is an identity element at $x \in P$, in which case the "difference" between $g_{1}^{\prime}$ and $g_{2}^{\prime}$ can be considered as a linear map from $T_{x} P$ to the fibre $A_{x}$ of the Lie algebroid, and this map (together with the zero map in the other direction) provides the required homotopy operator.

As a consequence of this representation up to homotopy, the representations of $J^{1} G$ on $A$ and $T P$ descend to induce honest representations of $G$ on the (generally singular) normal bundle $T P / \rho(A)$ to the orbits and isotropy bundle ker $\rho$ and on the determinant line bundle $Q_{A}$. In particular, the latter representation defines an element of first cohomology of $G$ with values in the multiplicative real numbers. This is the modular class of the groupoid.

In the situation of Example 3.11, where $A$ is an integrable subbundle of $T P$ we may take $G$ to be the holonomy groupoid of the foliation, in which case we recover the linearized holonomy representation of the foliation and the modular class of the foliation in groupoid cohomology discussed in $\mathrm{Ya}$ ] and Chapter IV of [M-S.

\section{References}

[B-B] Beilinson, A. and Bernstein, J., A proof of Jantzen conjectures, Advances in Soviet Mathematics 16 pt. 1 (1993), 1-50.

[Bo] Borel, A. et al., Algebraic D-modules, Academic Press, 1987.

[B-T] Bott, R. and Tu, L., Differential forms in algebraic topology, Springer-Verlag, 1982. 
[Br] Brylinski, J-L., A differential complex for Poisson manifolds, J. Diff. Geo. 28 (1988), $93-114$

[B-Z] Brylinski, J-L. and Zuckerman, G., The outer derivation of a complex Poisson manifold, preprint, 1996.

[G-H] Griffiths, P. and Harris, J., Principles of algebraic geometry, Wiley-Interscience, 1987.

[K-T] Kamber, F. and Tondeur, P., Invariant differential operators and the cohomology of Lie algebra sheaves, Mem. AMS, no. 113, 1971.

[Kp] Knapp, A., Lie groups, Lie algebras, and cohomology, Princeton University Press, 1988.

[Ks] Kontsevich, M., Course on deformation theory, University of California, Berkeley, 1994.

[Kz] Koszul, J. L., Crochet de Schouten-Nijenhuis et cohomologie, Astérisque, hors serie, (1985), $257-271$.

[L-M] Lada, T. and Markl, M., Strongly homotopy Lie algebras, Comm. in Alg. 23 (1995), $2147-2161$.

[L-W] Lu, J. H. and Weinstein, A., Poisson Lie groups, dressing transformations, and Bruhat decompositions, Journal of Differential Geometry 31 (1990), 501 - 526.

[Lu] Lu, J. H., Multiplicative and affine Poisson structures on Lie groups, PhD thesis, University of California, Berkeley, (1990).

[Ma] Mackenzie, K., Lie groupoids and Lie algebroids in differential geometry, Cambridge University Press, 1987.

[M-S] Moore, C. and Schochet, C., Global Analysis on Foliated Spaces, MSRI Publications 9, Springer-Verlag, New York (1988).

[STS] Semenov-Tian-Shansky, M. A., Dressing transformations and Poisson Lie group actions, Publ. RIMS, Kyoto University 21 (1985), 1237 - 1260.

[We] Weinstein, A., The modular automorphism group of a Poisson manifold, preprint, 1996. 
$[\mathrm{Xu}] \mathrm{Xu}, \mathrm{P} .$, Gerstenhaber algebras and BV-algebras in Poisson geometry, preprint, 1996.

[Ya] Yamagami, S., Modular cohomology class of foliation and Takesaki's duality, Geometric methods in operator algebras (Kyoto, 1983), Pitman Res. Notes Math. Ser., v. 123, Longman Sci. Tech., Harlow, 1986, pp. 415-439. 